TRANSACTIONS OF THE

AMERICAN MATHEMATICAL SOCIETY

Volume 359, Number 9, September 2007, Pages 4259-4284

S 0002-9947(07)04148-7

Article electronically published on April 11, 2007

\title{
ROOT NUMBERS OF ABELIAN VARIETIES
}

\author{
MARIA SABITOVA
}

\begin{abstract}
We generalize a theorem of D. Rohrlich concerning root numbers of elliptic curves over number fields. Our result applies to arbitrary abelian varieties. Namely, under certain conditions which naturally extend the conditions used by $\mathrm{D}$. Rohrlich, we show that the root number $W(A, \tau)$ associated to an abelian variety $A$ over a number field $F$ and a complex finite-dimensional irreducible representation $\tau$ of $\operatorname{Gal}(\bar{F} / F)$ with real-valued character is equal to 1 . We also show that our result is consistent with a refined version of the conjecture of Birch and Swinnerton-Dyer.
\end{abstract}

\section{INTRODUCTION}

The main object of study in this paper is the root number $W(A, \tau)$ associated to an abelian variety $A$ of dimension $g$ over a number field $F$ and a continuous irreducible complex finite-dimensional representation $\tau$ of $\operatorname{Gal}(\bar{F} / F)$ with real-valued character. The root number $W(A, \tau)$ is a complex number of absolute value 1 . The conjectural equation for the twisted $L$-function $L(A, \tau, s)$ associated to $A$ and $\tau$ implies

$$
W(A, \tau)=(-1)^{\operatorname{ord}_{s=1} L(A, \tau, s)} .
$$

In this paper we generalize a result by D. Rohrlich for elliptic curves ( 7 , p. 313, Prop. E) to abelian varieties. We prove the following theorem:

Theorem 0.1. Let $F$ be a number field, $L$ a finite Galois extension of $F$, and $\tau$ an irreducible complex finite-dimensional representation of $\operatorname{Gal}(L / F)$ with realvalued character. Let $g$ be a fixed positive integer and assume that the decomposition subgroups of $\operatorname{Gal}(L / F)$ at all the places of $F$ lying over all the primes less than or equal to $2 g+1$ are abelian. If the Schur index $m_{\mathbb{Q}}(\tau)$ is 2 , then $W(A, \tau)=1$ for every abelian variety $A$ of dimension $g$ over $F$.

Theorem 0.1 is predicted by the conjectures of Birch-Swinnerton-Dyer and Deligne-Gross. Namely, the conjectures of Birch-Swinnerton-Dyer and DeligneGross imply

$$
\operatorname{ord}_{s=1} L(A, \tau, s)=\left\langle\sigma_{A}, \tau\right\rangle,
$$

where $\sigma_{A}$ is the natural representation of $\operatorname{Gal}(\bar{F} / F)$ on $\mathbb{C} \otimes_{\mathbb{Z}} A(\bar{F})$ and $\left\langle\sigma_{A}, \tau\right\rangle$ is the multiplicity of $\tau$ in $\sigma_{A}$ ([5, p. 127, Prop. 2). Thus, we get from (0.1) and (0.2):

$$
W(A, \tau)=(-1)^{\left\langle\sigma_{A}, \tau\right\rangle} .
$$

Received by the editors May 6, 2005 and, in revised form, July 21, 2005.

2000 Mathematics Subject Classification. Primary 11G10; Secondary 11F80, 11G40, 11R32.

Key words and phrases. Abelian variety, root number, Weil-Deligne group. 
Since $\sigma_{A}$ is realizable over $\mathbb{Q}$ and $\tau$ is irreducible, $m_{\mathbb{Q}}(\tau)$ divides $\left\langle\sigma_{A}, \tau\right\rangle$. Thus, if $m_{\mathbb{Q}}(\tau)=2$, then $W(A, \tau)=1$ for every abelian variety $A$ over $F$ if $(0.2)$ is true (cf. 7], p. 313).

To prove Theorem 0.1 we use the following formula:

$$
W(A, \tau)=\prod_{v} W\left(A_{v}, \tau_{v}\right),
$$

where $v$ runs through all the places of $F, A_{v}=A \times{ }_{F} F_{v}, F_{v}$ denotes the completion of $F$ with respect to $v$, and $\tau_{v}$ is the restriction of $\tau$ to $\operatorname{Gal}\left(\overline{F_{v}} / F_{v}\right) \hookrightarrow \operatorname{Gal}(\bar{F} / F)$. To define $W\left(A_{v}, \tau_{v}\right)$ for every place $v$ let $\sigma_{v}^{\prime}$ denote the representation of the Weil-Deligne group $\mathcal{W}^{\prime}\left(\bar{F}_{v} / F_{v}\right)$ associated to the first cohomology of $A_{v}$. Then $W\left(A_{v}, \tau_{v}\right)=W\left(\sigma_{v}^{\prime} \otimes \tau_{v}\right)$, where $\tau_{v}$ is viewed as a representation of $\mathcal{W}^{\prime}\left(\bar{F}_{v} / F_{v}\right)$. We will in fact show the following stronger result:

Theorem 0.2. $W\left(A_{v}, \tau_{v}\right)=1$ for all $v$ under the hypotheses of Theorem 0.1 ,

First, we describe $W\left(A_{v}, \tau_{v}\right)$. If $v$ is an infinite place, then $\sigma_{v}^{\prime}$ is associated to the components of $H^{1}\left(A_{v}(\mathbb{C}), \mathbb{C}\right)$ in the Hodge decomposition. We show in Lemma 2.1 that

$$
W\left(A_{v}, \tau_{v}\right)=(-1)^{g \operatorname{dim} \tau} .
$$

If $v$ is a finite place, then

$$
W\left(\sigma_{v}^{\prime} \otimes \tau_{v}\right)=\frac{\epsilon\left(\sigma_{v}^{\prime} \otimes \tau_{v}, \psi_{v}, d x_{v}\right)}{\left|\epsilon\left(\sigma_{v}^{\prime} \otimes \tau_{v}, \psi_{v}, d x_{v}\right)\right|},
$$

where $\psi_{v}$ is a nontrivial additive character of $F_{v}$ and $d x_{v}$ is a Haar measure on $F_{v}$. Here $\sigma_{v}^{\prime}$ is isomorphic to the representation of $\mathcal{W}^{\prime}\left(\bar{F}_{v} / F_{v}\right)$ afforded by $H_{l}^{1}\left(A_{v}\right)$, where $l$ is a rational prime different from the residual characteristic of $F_{v}$. It is known that $H_{l}^{1}\left(A_{v}\right) \cong V_{l}\left(A_{v}\right)^{*}$ as $\operatorname{Gal}\left(\bar{F}_{v} / F_{v}\right)$-modules over $\mathbb{Q}_{l}$, where $V_{l}\left(A_{v}\right)=$ $T_{l}\left(A_{v}\right) \otimes_{\mathbb{Z}_{l}} \mathbb{Q}_{l}, T_{l}\left(A_{v}\right)$ is the $l$-adic Tate module of $A_{v}$, and $V_{l}\left(A_{v}\right)^{*}$ denotes the contragredient of $V_{l}\left(A_{v}\right)$. Thus, we can assume that $\sigma_{v}^{\prime}$ is the representation of $\mathcal{W}^{\prime}\left(\bar{F}_{v} / F_{v}\right)$ associated to $V_{l}\left(A_{v}\right)^{*}$. Clearly, $W\left(\sigma_{v}^{\prime} \otimes \tau_{v}\right)$ does not depend on the choice of $d x_{v}$, and it turns out that $W\left(\sigma_{v}^{\prime} \otimes \tau_{v}\right)$ does not depend on the choice of $\psi_{v}$ either. Moreover, $W\left(\sigma_{v}^{\prime} \otimes \tau_{v}\right)= \pm 1$ (see Subsection 1.1).

We consider two cases: $A_{v}$ is an abelian variety with potential good reduction, and the general case. If $A_{v}$ has potential good reduction, it follows from the criterion of Néron-Ogg-Shafarevič that $\sigma_{v}^{\prime}$ is actually a representation of the Weil group $\mathcal{W}\left(\bar{F}_{v} / F_{v}\right)$. If the characteristic of the residue class field $k_{v}$ of $F_{v}$ is greater than $2 g+1$, we use the theory of Serre-Tate together with methods of the representation theory to describe the class of $\sigma_{v}^{\prime} \otimes \omega_{v}^{1 / 2}$ in the Grothendieck group of virtual representations of $\mathcal{W}\left(\bar{F}_{v} / F_{v}\right)$ (Corollary 1.7, Formula (1.6) ). Here $\omega_{v}$ is the onedimensional representation of $\mathcal{W}\left(\bar{F}_{v} / F_{v}\right)$ given by

$$
\left.\omega_{v}\right|_{I_{v}}=1, \quad \omega_{v}\left(\Phi_{v}\right)=q_{v}^{-1}
$$

where $I_{v}$ is the inertia subgroup of $\operatorname{Gal}\left(\bar{F}_{v} / F_{v}\right), \Phi_{v}$ is an inverse Frobenius element of $\operatorname{Gal}\left(\bar{F}_{v} / F_{v}\right)$, and $q_{v}=\operatorname{card}\left(k_{v}\right)$. Since the root number of representations of $\mathcal{W}\left(\bar{F}_{v} / F_{v}\right)$ is multiplicative in short exact sequences, this result enables us to prove the following formula for $W\left(\sigma_{v}^{\prime} \otimes \tau_{v}\right)$ when $\operatorname{char}\left(k_{v}\right)>2 g+1$ (cf. Proposition [1.9) :

$$
W\left(\sigma_{v}^{\prime} \otimes \tau_{v}\right)=\operatorname{det} \tau_{v}(-1)^{l_{1}} \cdot \beta^{\operatorname{dim} \tau} \cdot \gamma^{l_{2}} \cdot(-1)^{\left\langle\nu_{v}, \tau_{v}\right\rangle},
$$


where $l_{1} \in \mathbb{Z}, \beta= \pm 1, \gamma= \pm 1, l_{2}=\left\langle 1, \tau_{v}\right\rangle+\left\langle\eta_{v}, \tau_{v}\right\rangle, \eta_{v}$ is the unramified quadratic character of $F_{v}^{\times}$, and $\nu_{v}$ is a representation of $\operatorname{Gal}\left(\bar{F}_{v} / F_{v}\right)$ realizable over $\mathbb{Q}$ (cf. [7, p. 318, Thm. 1).

In the general case we use the theory of uniformization of abelian varieties ([2]). According to this theory there exists a semi-abelian variety $G_{v}$ over $F_{v}$ and a discrete subgroup $Y_{v}$ of $G_{v}$ such that, in terms of rigid geometry, $A_{v}$ is isomorphic to the quotient $G_{v} / Y_{v}$. The semi-abelian variety $G_{v}$ fits into an exact sequence

$$
0 \longrightarrow T_{v} \longrightarrow G_{v} \longrightarrow B_{v} \longrightarrow 0
$$

where $B_{v}$ is an abelian variety over $F_{v}$ with potential good reduction, and $T_{v}$ is a torus over $F_{v}$ of dimension $r$; $Y_{v}$ is an étale sheaf of free abelian groups over $\operatorname{Spec}\left(F_{v}\right)$ of rank $r$. To describe $\sigma_{v}^{\prime}$ in this case we use a formula of M. Raynaud (44, p. 314) which gives the action of the inertia group $I_{v}$ on the $l^{n}$-torsion points of an abelian variety over a non-Archimedean local field in the case when the uniformization data splits. We need this formula to show that in this case

$$
\sigma_{v}^{\prime} \cong \kappa_{v} \oplus\left(\chi_{v} \otimes \omega_{v}^{-1} \otimes \operatorname{sp}(2)\right)
$$

where $\kappa_{v}$ is the representation of $\mathcal{W}^{\prime}\left(\bar{F}_{v} / F_{v}\right)$ associated to the natural $l$-adic representation of $\operatorname{Gal}\left(\bar{F}_{v} / F_{v}\right)$ on $V_{l}\left(B_{v}\right)^{*}$,

$$
\chi_{v}: \operatorname{Gal}\left(\bar{F}_{v} / F_{v}\right) \longrightarrow \mathrm{GL}_{r}(\mathbb{Z})
$$

is the representation of $\operatorname{Gal}\left(\bar{F}_{v} / F_{v}\right)$ corresponding to the Galois module $Y_{v}\left(\bar{F}_{v}\right)$, and $\operatorname{sp}(2)$ is given by (1.1) (see Proposition 1.10). Since the root number of a direct sum of representations of $\mathcal{W}^{\prime}\left(\bar{F}_{v} / F_{v}\right)$ equals the product of the root numbers of the summands, we get from (0.5)

$$
W\left(\sigma_{v}^{\prime} \otimes \tau_{v}\right)=W\left(\kappa_{v} \otimes \tau_{v}\right) \cdot W\left(\chi_{v} \otimes \omega_{v}^{-1} \otimes \tau_{v} \otimes \operatorname{sp}(2)\right) .
$$

If $\operatorname{char}\left(k_{v}\right)>2 g+1$, then (0.4) can be applied to $\kappa_{v}$, i.e.,

$$
W\left(\kappa_{v} \otimes \tau_{v}\right)=\operatorname{det} \tau_{v}(-1)^{l_{1}} \cdot \beta^{\operatorname{dim} \tau} \cdot \gamma^{l_{2}} \cdot(-1)^{\left\langle\nu_{v}, \tau_{v}\right\rangle},
$$

where $l_{1}, \beta, \gamma, l_{2}$, and $\nu_{v}$ are as in (0.4) when $\sigma_{v}^{\prime}$ is replaced by $\kappa_{v}$.

The rest of the proof of Theorem 0.2 is analogous to one of Proposition E ([7], p. 347). Namely, it follows from the Lemma on p. 339 and the Lemma on p. 347 in [7] that $\operatorname{dim} \tau$ is even. Hence we get from (0.3) that $W\left(A_{v}, \tau_{v}\right)=1$ for infinite places. If $v$ is a finite place, then the assumption $m_{\mathbb{Q}}(\tau)=2$ implies $W\left(\chi_{v} \otimes \omega_{v}^{-1} \otimes \tau_{v} \otimes \operatorname{sp}(2)\right)=$ 1 , hence we have from 0.6

$$
W\left(\sigma_{v}^{\prime} \otimes \tau_{v}\right)=W\left(\kappa_{v} \otimes \tau_{v}\right)
$$

If $v$ is a finite place such that $\operatorname{char}\left(k_{v}\right)>2 g+1$, then (0.7) holds which, together with the assumption $m_{\mathbb{Q}}(\tau)=2$, implies $W\left(\kappa_{v} \otimes \tau_{v}\right)=1$, hence $W\left(\sigma_{v}^{\prime} \otimes \tau_{v}\right)=1$.

If $v$ is a finite place such that $\operatorname{char}\left(k_{v}\right) \leq 2 g+1$, then the conditions on bad primes in Theorem 0.1 imply that $\tau_{v}$ is symplectic ([7], Lemma on p. 347). Also, $\kappa_{v} \otimes \omega_{v}^{1 / 2}$ is symplectic, because $\kappa_{v}$ comes from an abelian variety (see Subsection 1.1). Since real powers of $\omega_{v}$ do not change the root number,

$$
W\left(\kappa_{v} \otimes \tau_{v}\right)=W\left(\kappa_{v} \otimes \omega_{v}^{1 / 2} \otimes \tau_{v}\right),
$$

where $W\left(\kappa_{v} \otimes \omega_{v}^{1 / 2} \otimes \tau_{v}\right)=1$, as the root number of the tensor product of two symplectic representations of $\mathcal{W}\left(\bar{F}_{v} / F_{v}\right)$ is 1 ([7], p. 319, Prop. 2 and the remark after it). Thus, in this case we also have $W\left(\sigma_{v}^{\prime} \otimes \tau_{v}\right)=1$ by $(\underline{0.8)}$ and (0.9). 
This paper is organized in the following way. In Section 1 we study the root number $W\left(\sigma^{\prime} \otimes \tau\right)$, where $\tau$ is a complex finite-dimensional representation of $\operatorname{Gal}(\bar{K} / K)$ with real-valued character, $K$ is a local non-Archimedean field of characteristic zero, and $\sigma^{\prime}$ is the representation of $\mathcal{W}^{\prime}(\bar{K} / K)$ associated to the natural $l$-adic representation of $\operatorname{Gal}(\bar{K} / K)$ on $V_{l}(A)^{*}$, where $A$ is an abelian variety over $K$. Subsection 1.1 contains general facts and notation used in the paper. In Subsection 1.2 we study the case of an abelian variety with potential good reduction. Subsection 1.3 deals with the general case. In Section 2 we give the proof of Theorem 0.2 . We put proofs of the results of Subsection 1.2 in Appendix A. Appendix B contains two lemmas needed for the proof of the main result of Subsection 1.3 (Proposition 1.10).

Unless stated otherwise, we assume that all the representations under consideration are complex and finite-dimensional.

\section{LOCAL CASE}

1.1. General facts and notation. Let $K$ be a non-Archimedean local field of characteristic zero with ring of integers $\mathcal{O}$ and residue class field $k$ of characteristic $p$. Let $\bar{K}$ be a fixed algebraic closure of $K$ and let $K^{u n r}$ be the maximal unramified extension of $K$ contained in $\bar{K}$. Let $I=\mathrm{Gal}\left(\bar{K} / K^{u n r}\right)$ be the inertia subgroup of $\operatorname{Gal}(\bar{K} / K)$ and let $\Phi$ be an inverse Frobenius element of $\operatorname{Gal}(\bar{K} / K)$, i.e., $\Phi$ is a preimage of the inverse of the Frobenius automorphism under the decomposition map

$$
\pi: \operatorname{Gal}(\bar{K} / K) \longrightarrow \operatorname{Gal}(\bar{k} / k) .
$$

Let $\mathcal{W}(\bar{K} / K)$ denote the Weil group of $K([6, \S 1)$. By a representation $\sigma$ of $\mathcal{W}(\bar{K} / K)$ we mean a continuous homomorphism

$$
\sigma: \mathcal{W}(\bar{K} / K) \longrightarrow \mathrm{GL}(U),
$$

where $U$ is a finite-dimensional complex vector space. Let $\omega: \mathcal{W}(\bar{K} / K) \longrightarrow \mathbb{C}^{\times}$be the one-dimensional representation of $\mathcal{W}(\bar{K} / K)$ given by

$$
\left.\omega\right|_{I}=1, \quad \omega(\Phi)=q^{-1},
$$

where $q=\operatorname{card}(k)$. For a finite extension $F$ of $K$ contained in $\bar{K}$, we identify by local class field theory the one-dimensional representations of $\mathcal{W}(\bar{K} / F)$ with characters of $F^{\times}$(i.e., continuous homomorphisms from $F^{\times}$into $\left.\mathbb{C}^{\times}\right)$. Also, if $\phi$ is a representation of $\mathcal{W}(\bar{K} / F)$, the representation of $\mathcal{W}(\bar{K} / K)$ induced by $\phi$ will be denoted by $\operatorname{Ind}_{K}^{F} \phi$. Analogously, if $\psi$ is a representation of $\mathcal{W}(\bar{K} / K)$, then the restriction of $\psi$ to $\mathcal{W}(\bar{K} / F)$ will be denoted by $\operatorname{Res}_{K}^{F} \psi$.

Let $\mathcal{W}^{\prime}(\bar{K} / K)$ denote the Weil-Deligne group of $K([\underline{6}, \S 3)$. By a representation $\sigma^{\prime}$ of $\mathcal{W}^{\prime}(\bar{K} / K)$ we mean a continuous homomorphism

$$
\sigma^{\prime}: \mathcal{W}^{\prime}(\bar{K} / K) \longrightarrow \mathrm{GL}(U),
$$

where $U$ is a finite-dimensional complex vector space and the restriction of $\sigma^{\prime}$ to the subgroup $\mathbb{C}$ of $\mathcal{W}^{\prime}(\bar{K} / K)$ is complex analytic. It is known that there is a bijection between representations of $\mathcal{W}^{\prime}(\bar{K} / K)$ and pairs $(\sigma, N)$, where $\sigma: \mathcal{W}(\bar{K} / K) \longrightarrow$ $\mathrm{GL}(U)$ is a representation of $\mathcal{W}(\bar{K} / K)$ and $N$ is a nilpotent endomorphism on $U$ such that

$$
\sigma(g) N \sigma(g)^{-1}=\omega(g) N, \quad g \in \mathcal{W}(\bar{K} / K) .
$$


In what follows we identify $\sigma^{\prime}$ with the corresponding pair $(\sigma, N)$ and write $\sigma^{\prime}=$ $(\sigma, N)$. Also, a representation $\sigma$ of $\mathcal{W}(\bar{K} / K)$ is identified with the representation $(\sigma, 0)$ of $\mathcal{W}^{\prime}(\bar{K} / K)([\underline{6}, \S \S 1-3)$.

For a positive integer $n$ let $\operatorname{sp}(n)=(\sigma, N)$ denote the special representation of dimension $n$, i.e., the representation of $\mathcal{W}^{\prime}(\bar{K} / K)$ on $\mathbb{C}^{n}$ (with the standard basis $\left.e_{0}, \ldots, e_{n-1}\right)$ given by the following formulas:

$$
\begin{array}{rll}
\sigma(g) e_{i} & =\omega(g)^{i} e_{i}, & 0 \leq i \leq n-1, g \in \mathcal{W}(\bar{K} / K), \\
N e_{j} & =e_{j+1}, & \\
N e_{n-1} & =0 . &
\end{array}
$$

We say that a representation $\sigma^{\prime}=(\sigma, N)$ of $\mathcal{W}^{\prime}(\bar{K} / K)$ is admissible if $\sigma$ is semisimple $([\underline{6}$, p. $132, \S 5)$.

Let $A$ be an abelian variety over $K$. For a rational prime $l$ different from $p=$ $\operatorname{char}(k)$ let $T_{l}(A)$ be the $l$-adic Tate module of $A$. It is a free $\mathbb{Z}_{l}$-module of rank $2 g$, where $g=\operatorname{dim} A$. Put $V_{l}(A)=T_{l}(A) \otimes_{\mathbb{Z}_{l}} \mathbb{Q}_{l}$ and let

$$
\sigma_{l}: \operatorname{Gal}(\bar{K} / K) \longrightarrow \operatorname{GL}\left(V_{l}(A)^{*}\right)
$$

denote the contragredient of the natural $l$-adic representation of $\operatorname{Gal}(\bar{K} / K)$ on $V_{l}(A)$. We are interested in the representation $\sigma^{\prime}=(\sigma, N)$ of $\mathcal{W}^{\prime}(\bar{K} / K)$ associated to $\sigma_{l}$ by the standard procedure (see e.g., [6], $\S 4$ ). Let $\imath: \mathbb{Q}_{l} \hookrightarrow \mathbb{C}$ be a field embedding. Then $\sigma: \mathcal{W}(\bar{K} / K) \longrightarrow \mathrm{GL}\left(V_{l}(A)^{*} \otimes_{\imath} \mathbb{C}\right)$ is a representation of $\mathcal{W}(\bar{K} / K)$ (which is not necessarily obtained from the restriction of $\sigma_{l}$ to $\mathcal{W}(\bar{K} / K)$ by extending scalars via $\left.\imath: \mathbb{Q}_{l} \hookrightarrow \mathbb{C}\right)$ and $N \in \operatorname{End}\left(V_{l}(A)^{*} \otimes_{\imath} \mathbb{C}\right)$ is a nilpotent endomorphism (see [6], p. 130, $\S 4$ for more details). A priori, $\sigma^{\prime}$ depends on the choice of $l$ and $\imath$, but by abuse of notation we write $\sigma^{\prime}$ instead of $\sigma_{l, 2}^{\prime}$. We will prove later that in our context $\sigma^{\prime}$ does not depend on the choice of $l$ and $\imath$. Let $\tau$ be a representation of $\operatorname{Gal}(\bar{K} / K)$ with real-valued character. Our goal in this section is to compute the root number $W\left(\sigma^{\prime} \otimes \tau\right)$.

Note that there is a nondegenerate, skew-symmetric, $\operatorname{Gal}(\bar{K} / K)$-equivariant pairing

$$
\langle-,-\rangle: V_{l}(A) \times V_{l}(A) \longrightarrow \mathbb{Q}_{l} \otimes \omega_{l},
$$

where $\omega_{l}$ is the $l$-adic cyclotomic character of $\operatorname{Gal}(\bar{K} / K)$. Indeed, let $A^{\vee}$ be the dual abelian variety to $A$ and let

$$
e_{l}: T_{l}(A) \times T_{l}\left(A^{\vee}\right) \longrightarrow \mathbb{Z}_{l} \otimes \omega_{l}
$$

be the Weil pairing, which is nondegenerate and $\operatorname{Gal}(\bar{K} / K)$-equivariant ([3], p. $131, \S 16)$. Let $\mathcal{L}$ be an ample invertible sheaf on $A(\underline{3}$, p. 114, Cor. 7.2). Then $\varphi_{\mathcal{L}}: A \longrightarrow A^{\vee}$ is an isogeny $([3]$, p. $119, \S 10)$, and the pairing

$$
e_{l}^{\mathcal{L}}: T_{l}(A) \times T_{l}(A) \longrightarrow \mathbb{Z}_{l} \otimes \omega_{l}
$$

defined for $a, a^{\prime} \in T_{l}(A)$ by $e_{l}^{\mathcal{L}}\left(a, a^{\prime}\right)=e_{l}\left(a, \varphi_{\mathcal{L}}\left(a^{\prime}\right)\right)$ is skew-symmetric ([3], p. 134, Prop. 16.6). It is easy to show that the pairing on $V_{l}(A)$ obtained from $e_{l}^{\mathcal{L}}$ by extending scalars to $\mathbb{Q}_{l}$ is nondegenerate and $\operatorname{Gal}(\bar{K} / K)$-equivariant.

Having $\langle-,-\rangle$, it is easy to show that $\sigma^{\prime} \otimes \omega^{1 / 2}$ is symplectic (cf. [6], p. 150, $\S 16)$. Then $\sigma^{\prime} \otimes \omega^{1 / 2} \otimes \tau$ is self-contragredient and of trivial determinant, hence $W\left(\sigma^{\prime} \otimes \omega^{1 / 2} \otimes \tau\right)$ does not depend on the choice of a nontrivial additive character of $K$ and $W\left(\sigma^{\prime} \otimes \omega^{1 / 2} \otimes \tau\right)= \pm 1\left(\left[7\right.\right.$, p. 315). Since $W\left(\sigma^{\prime} \otimes \tau\right)=W\left(\sigma^{\prime} \otimes \omega^{1 / 2} \otimes \tau\right)$, the same conclusion holds for $W\left(\sigma^{\prime} \otimes \tau\right)$. 
One of the main theories we are using to find a formula for $W\left(\sigma^{\prime} \otimes \tau\right)$ is the theory of uniformization of abelian varieties. According to this theory there exists a semi-abelian variety $G$ over $K$ and a discrete subgroup $Y$ of $G$ such that, in terms of rigid geometry, $A$ is isomorphic to the quotient $G / Y$. The semi-abelian variety $G$ fits into an exact sequence

$$
0 \longrightarrow T \longrightarrow G \longrightarrow B \longrightarrow 0
$$

where $B$ is an abelian variety over $K$ with potential good reduction, and $T$ is a torus over $K$ of dimension $r$; $Y$ is an étale sheaf of free abelian groups over $\operatorname{Spec}(K)$ of rank $r$.

1.2. Case of an abelian variety with potential good reduction. We keep the notation of Subsection 1.1. Let $B$ be an abelian variety over $K$ with potential good reduction and let

$$
\kappa_{l}: \operatorname{Gal}(\bar{K} / K) \longrightarrow \mathrm{GL}\left(V_{l}(B)^{*}\right)
$$

denote the natural $l$-adic representation of $\operatorname{Gal}(\bar{K} / K)$ on $V_{l}(B)^{*}$. First, note that the representation $\kappa^{\prime}=(\kappa, S)$ of $\mathcal{W}^{\prime}(\bar{K} / K)$ associated to $\kappa_{l}$ is actually a representation of $\mathcal{W}(\bar{K} / K)$, i.e., $S=0$. Indeed, $\kappa^{\prime}$ is a representation of $\mathcal{W}(\bar{K} / K)$ if and only if $\kappa_{l}$ is trivial on an open subgroup of $I$ ([6], p. 131, Prop.(i)). Let

$$
\psi_{l}: \operatorname{Gal}(\bar{K} / K) \longrightarrow \operatorname{Aut}\left(T_{l}(B)\right)
$$

denote the representation corresponding to the $\operatorname{Gal}(\bar{K} / K)$-module $T_{l}(B)$. Since $B$ has potential good reduction, the image by $\psi_{l}$ of $I$ is finite (9], p. 496, Thm. 2(i)), which implies that the image by $\kappa_{l}$ of $I$ is finite, hence $\kappa_{l}$ is trivial on an open subgroup of $I$ (cf. [6], p. 148).

Lemma 1.1. A complex finite-dimensional representation of a group is semisimple if and only if its restriction to a subgroup of finite index is semisimple.

Proof. It is known that a complex finite-dimensional representation $\lambda$ of a group is semisimple if and only if its restriction to a normal subgroup of finite index is semisimple ([1], p. 82, Prop. 1 and [6], p. 148). Moreover, since every subgroup of finite index contains a normal subgroup of finite index, this implies that $\lambda$ is semisimple if and only if its restriction to a subgroup of finite index is semisimple.

Lemma 1.2. $\kappa$ is semisimple.

Proof. Since the image by $\kappa$ of $I$ is finite, by Lemma 1.1 it is enough to show that $\kappa(\Phi)$ is diagonalizable. Also, if $L \subset \bar{K}$ is a finite extension of $K$ over which $B$ acquires good reduction, then $\kappa$ is semisimple if and only if its restriction to $\mathcal{W}(\bar{K} / L)$ is semisimple again by Lemma 1.1. Thus, we can assume that $B$ has good reduction (cf. [6], p. 148). Let $B_{0}$ be the Néron minimal model of $B$ and $\tilde{B}=B_{0} \times \mathcal{O}$ $k$ the special fiber of $B_{0}$. Since $B$ has good reduction, the reduction map defines a $\operatorname{Gal}(\bar{K} / K)$-equivariant isomorphism of $T_{l}(B)$ onto $T_{l}(\tilde{B})$, where $\operatorname{Gal}(\bar{K} / K)$ acts on $T_{l}(\tilde{B})$ via the decomposition map $\pi([9]$, p. 495, Lem. 2). Thus,

$$
V_{l}(B) \cong V_{l}(\tilde{B})
$$

as $\operatorname{Gal}(\bar{K} / K)$-modules. 
Lemma 1.3. Let $D$ be a group and let $U$ be a finite-dimensional representation of $D$ over a field $\ell$. Then $U$ is semisimple if the subalgebra of $\operatorname{End}_{\ell}(U)$ generated by the image of $D$ is semisimple.

Proof. Obvious.

Since the subalgebra of $\operatorname{End}_{\mathbb{Q}_{l}}\left(V_{l}(\tilde{B})\right)$ generated by the automorphisms of $V_{l}(\tilde{B})$ defined by elements of $\operatorname{Gal}(\bar{k} / k)$ is semisimple $([10]$, p. 138), the natural $l$-adic representation $\beta_{l}$ of $\operatorname{Gal}(\bar{k} / k)$ on $V_{l}(\tilde{B})$ is semisimple by Lemma 1.3. Since $\operatorname{Gal}(\bar{k} / k)$ is abelian, $\beta_{l}$ is a direct sum of one-dimensional representations, hence $\beta_{l}(\pi(\Phi))$ is diagonalizable, consequently, $\kappa_{l}^{*}(\Phi)$ is diagonalizable, because $\kappa_{l}^{*}(\Phi)$ is equivalent to $\beta_{l}(\pi(\Phi))$ via (1.3). This proves that $\kappa(\Phi)$ is diagonalizable, because $\kappa(\Phi)$ is just $\kappa_{l}(\Phi)$ considered as an element of $\operatorname{GL}\left(V_{l}(B)^{*} \otimes_{\imath} \mathbb{C}\right)$.

Corollary 1.4. The representation $\kappa$ does not depend on the choice of $l$ and $\imath$.

Proof. [6], p. 148 and Lemma 1.2 .

Since $B$ has potential good reduction, by the theory of Serre-Tate there exists a minimal finite subextension $L / K^{u n r}$ of $\bar{K} / K^{u n r}$ over which $B$ acquires good reduction. It is a Galois extension and it is tamely ramified if $p>2 m+1$, where $m=\operatorname{dim} B$. Moreover, $\operatorname{Gal}(\bar{K} / L)$ is contained in the kernel of the representation $\psi_{l}$ ([9], p. 497, Cor. 2 and p. 498, Cor. 3). Thus $\kappa$, and consequently $\kappa \otimes \omega^{1 / 2}$, can be considered as representations of the group

$$
\mathcal{W}(L / K)=\mathcal{W}(\bar{K} / K) / \operatorname{Gal}(\bar{K} / L) \cong \operatorname{Gal}\left(L / K^{u n r}\right) \rtimes\langle\Phi\rangle,
$$

where $\langle\Phi\rangle$ is the infinite cyclic group generated by $\Phi$ (cf. [7, p. 331). Throughout this subsection we assume that $p>2 m+1$. Then, under this assumption $E=$ $\operatorname{Gal}\left(L / K^{u n r}\right)$ is a finite cyclic group of order not divisible by $p$ and $\kappa \otimes \omega^{1 / 2}$ is a semisimple (by Lemma 1.2), symplectic (see Subsection 1.1) representation of the semi-direct product $G=E \rtimes\langle\Phi\rangle$ of finite and infinite cyclic groups. Using the Corollary on p. 499 in $[9$, it is immediate that $\kappa$ has $\mathbb{Q}$-valued character. Since $\omega$ is trivial on $I$, it follows that $\operatorname{Res}_{E}^{G}\left(\kappa \otimes \omega^{1 / 2}\right)$ has $\mathbb{Q}$-valued character. The following results give a description of such a representation, i.e., a semisimple symplectic representation $\lambda$ of a semi-direct product of a finite cyclic group $E$ and an infinite cyclic group such that the restriction of $\lambda$ to $E$ has $\mathbb{Q}$-valued character. They will be used later to generalize a formula for the root number obtained by D. Rohrlich.

Proposition 1.5. Let $C=\langle c\rangle$ be an infinite cyclic group generated by an element $c$ and let $E=\langle e\rangle$ be a finite cyclic group of order $n$ generated by an element $e$. Let $G=E \rtimes C$ be a semi-direct product, where $C$ acts on $E$ via $c^{-1} e c=e^{k}$ for some $k \in(\mathbb{Z} / n \mathbb{Z})^{\times}$. Denote by $s$ the order of $k$ in $(\mathbb{Z} / n \mathbb{Z})^{\times}$. Then every irreducible symplectic representation $\lambda$ of $G$ factors through the group $H=G /\left\langle c^{2 s}\right\rangle$ and as a representation of $H$ has the form

$$
\lambda=\operatorname{Ind}_{E \rtimes \Gamma}^{H} \phi,
$$

where $\Gamma$ is a subgroup of $C /\left\langle c^{2 s}\right\rangle$ generated by an element $c^{x}$ and $\phi$ is a onedimensional representation of $E \rtimes \Gamma$ satisfying the following conditions:

(1) $\phi(e)=\xi$ for an $n$-th root of unity $\xi$ of order $d(d \neq 1,2)$,

(2) $x$ is the order of $k$ in $(\mathbb{Z} / d \mathbb{Z})^{\times}$,

(3) $x$ is even, 
(4) $\phi\left(c^{x}\right)=-1$,

(5) $1+k^{\frac{x}{2}} \equiv 0(\bmod d)$.

Conversely, every representation of this form is symplectic and irreducible.

Proof. See Appendix A.

In the notation of Proposition 1.5 let $\lambda=\operatorname{Infl}_{H}^{G} \operatorname{Ind}_{E \rtimes \Gamma}^{H} \phi$ be a symplectic irreducible representation of $G$ and $\theta$ the one-dimensional representation of $E \rtimes \Gamma$ such that $\theta\left(c^{x}\right)=-1, \theta(e)=1$. Let

$$
\hat{\lambda}=\operatorname{Infl}_{H}^{G} \operatorname{Ind}_{E \rtimes \Gamma}^{H}(\phi \otimes \theta) .
$$

Whereas $\lambda$ is symplectic, $\hat{\lambda}$ is realizable over $\mathbb{R}$, as can be checked using Proposition 39 ([8], p. 109).

For a group $D$ let $R(D)$ denote the Grothendieck group of the abelian category of finite-dimensional representations of $D$ over $\mathbb{C}$. If $\rho$ is such a representation we denote by $[\rho]$ the corresponding element of $R(D)$.

Proposition 1.6. Let $G=E \rtimes C$ be a semi-direct product as in Proposition 1.5 and $\lambda$ a semisimple symplectic representation of $G$. If $\operatorname{Res}_{E}^{G} \lambda$ has $\mathbb{Q}$-valued character, then in $R(G)$ we have

$$
[\lambda]=[\mu]+\left[\mu^{*}\right]+2 \cdot\left(\left[\mu_{0}\right]-\left[\mu_{0}^{\prime}\right]\right)+\left[\mu_{1}\right]+\cdots+\left[\mu_{a}\right],
$$

where $\mu$ is a representation of $G, \mu^{*}$ is the contragredient of $\mu, \mu_{0}$ and $\mu_{0}^{\prime}$ are symplectic representations of $G$ with finite images, $\mu_{1}, \ldots, \mu_{a}$ are irreducible symplectic subrepresentations of $\lambda$, and $\hat{\mu}_{1}, \ldots, \hat{\mu}_{a}$ are representations given by (1.4) such that $\hat{\mu}_{1} \oplus \cdots \oplus \hat{\mu}_{a}$ is realizable over $\mathbb{Q}$.

Proof. See Appendix A.

Corollary 1.7. Let $\kappa$ be the representation of $\mathcal{W}(\bar{K} / K)$ corresponding to $V_{l}(B)^{*}$, $m=\operatorname{dim} B$, and $p>2 m+1$. Then in $R(\mathcal{W}(\bar{K} / K))$ we have

$$
\left[\kappa \otimes \omega^{1 / 2}\right]=[\mu]+\left[\mu^{*}\right]+2 \cdot\left(\left[\mu_{0}\right]-\left[\mu_{0}^{\prime}\right]\right)+\left[\mu_{1}\right]+\cdots+\left[\mu_{a}\right]
$$

where $\mu$ is a representation of $\mathcal{W}(\bar{K} / K), \mu^{*}$ is the contragredient of $\mu, \mu_{0}$ and $\mu_{0}^{\prime}$ are symplectic representations of $\mathcal{W}(\bar{K} / K)$ with finite images, $\mu_{1}, \ldots, \mu_{a}$ are irreducible symplectic subrepresentations of $\kappa \otimes \omega^{1 / 2}$, and $\hat{\mu}_{1}, \ldots, \hat{\mu}_{a}$ are representations given by (1.4) such that $\hat{\mu}_{1} \oplus \cdots \oplus \hat{\mu}_{a}$ is realizable over $\mathbb{Q}$.

Let $\tau$ be a representation of $\operatorname{Gal}(\bar{K} / K)$ with real-valued character. To compute the root number $W(\kappa \otimes \tau)$ we generalize the following result by D. Rohrlich ([7], p. 318 , Thm. 1) :

Theorem 1.8. Let $\lambda$ be a two-dimensional irreducible, symplectic representation of $\operatorname{Gal}(\bar{K} / K)$ of the form $\lambda=I n d_{K}^{H_{2}} \phi$, where $H_{2}$ is the unramified quadratic extension of $K$ and $\phi$ is a tame character of $H_{2}^{\times}$. Then

$$
W(\lambda \otimes \tau)=\operatorname{det} \tau(-1) \cdot \phi\left(u_{H_{2} / K}\right)^{\operatorname{dim} \tau} \cdot(-1)^{\langle 1, \tau\rangle+\langle\eta, \tau\rangle+\langle\hat{\lambda}, \tau\rangle},
$$

where $\eta$ is the unramified quadratic character of $K^{\times}, \hat{\lambda}=\operatorname{Ind}_{K}^{H_{2}}(\phi \otimes \theta)$ for the unramified quadratic character $\theta$ of $H_{2}^{\times}$, and $u_{H_{2} / K}$ is a unit in the ring of integers of $H_{2}$ such that $H_{2}=K\left(u_{H_{2} / K}\right)$ and $u_{H_{2} / K}^{2} \in K$. 
Proposition 1.9. Let $\kappa$ be the representation of $\mathcal{W}(\bar{K} / K)$ corresponding to $V_{l}(B)^{*}$, $m=\operatorname{dim} B$, and $p>2 m+1$. Let $\tau$ be a representation of $\operatorname{Gal}(\bar{K} / K)$ with realvalued character. In the notation of Corollary 1.7 we have

$$
W(\kappa \otimes \tau)=\operatorname{det} \mu(-1)^{\operatorname{dim} \tau} \cdot \operatorname{det} \tau(-1)^{l_{1}} \cdot \alpha^{\operatorname{dim} \tau} \cdot(-1)^{l_{2}},
$$

where $l_{1}=\operatorname{dim} \mu+\frac{1}{2}\left(\operatorname{dim} \mu_{1}+\cdots+\operatorname{dim} \mu_{a}\right), \alpha= \pm 1, l_{2}=a \cdot\langle 1, \tau\rangle+a \cdot\langle\eta, \tau\rangle+$ $\left\langle\hat{\mu}_{1} \oplus \cdots \oplus \hat{\mu}_{a}, \tau\right\rangle$, and $\eta$ is the unramified quadratic character of $K^{\times}$.

Proof. See Appendix A.

1.3. General case. We keep the notation of Subsection 1.1. Let $\sigma^{\prime}=(\sigma, N)$ be the representation of $\mathcal{W}^{\prime}(\bar{K} / K)$ associated to the natural $l$-adic representation of $\operatorname{Gal}(\bar{K} / K)$ on $V_{l}(A)^{*}, \kappa$ the representation of $\mathcal{W}^{\prime}(\bar{K} / K)$ associated to the natural $l$-adic representation of $\operatorname{Gal}(\bar{K} / K)$ on $V_{l}(B)^{*}$, and

$$
\chi: \operatorname{Gal}(\bar{K} / K) \longrightarrow \mathrm{GL}_{r}(\mathbb{Z})
$$

the representation corresponding to the $\mathrm{Gal}(\bar{K} / K)$-module $Y(\bar{K})$. It is known that there is a finite Galois extension $L \subset \bar{K}$ of $K$ such that $\mathrm{Gal}(\bar{K} / L)$ acts trivially on $Y(\bar{K})$, hence $\chi$ has finite image. Here $\kappa$ is actually a representation of $\mathcal{W}(\bar{K} / K)$ (see Subsection 1.2) and we identify $\kappa$ with the representation $(\kappa, 0)$ of $\mathcal{W}^{\prime}(\bar{K} / K)$. Also, we identify $\chi$ with the representation $\left(\operatorname{Res}_{\mathcal{W}(\bar{K} / K)}^{\operatorname{Gal}(\bar{K} / K)} \chi, 0\right)$ of $\mathcal{W}^{\prime}(\bar{K} / K)$.

The main result of this subsection is the following proposition:

\section{Proposition 1.10.}

$$
\sigma^{\prime} \cong \kappa \oplus\left(\chi \otimes \omega^{-1} \otimes \operatorname{sp}(2)\right) .
$$

To prove Proposition 1.10 we will need the following lemmas.

Lemma 1.11. Let $\lambda^{\prime}=(\lambda, R)$ be the representation of $\mathcal{W}^{\prime}(\bar{K} / K)$ associated to the natural l-adic representation of $\operatorname{Gal}(\bar{K} / K)$ on $V_{l}(T)^{*}$. Then $R=0$ and

$$
\lambda \cong \chi \otimes \omega^{-1} \text {. }
$$

Proof. Let $X$ be the character group of $T$. Then $T(\bar{K}) \cong \operatorname{Hom}_{\mathbb{Z}}\left(X(\bar{K}), \bar{K}^{\times}\right)$as $\operatorname{Gal}(\bar{K} / K)$-modules over $\mathbb{Z}$, hence we have the following sequence of isomorphisms of $\mathrm{Gal}(\bar{K} / K)$-modules:

$$
\begin{aligned}
V_{l}(T) & =T_{l}(T) \otimes_{\mathbb{Z}_{l}} \mathbb{Q}_{l} \cong \operatorname{Hom}_{\mathbb{Z}}\left(X(\bar{K}), T_{l}\left(\bar{K}^{\times}\right)\right) \otimes_{\mathbb{Z}_{l}} \mathbb{Q}_{l} \\
& \cong\left(X(\bar{K}) \otimes_{\mathbb{Z}} \mathbb{Q}_{l}\right)^{*} \otimes_{\mathbb{Q}_{l}} V_{l}\left(\bar{K}^{\times}\right) .
\end{aligned}
$$

It is known that there is an injective homomorphism $\phi: Y \longrightarrow X$ with finite cokernel ([2], p. 58), consequently

$$
Y(\bar{K}) \otimes_{\mathbb{Z}} \mathbb{Q}_{l} \cong X(\bar{K}) \otimes_{\mathbb{Z}} \mathbb{Q}_{l}
$$

as $\operatorname{Gal}(\bar{K} / K)$-modules over $\mathbb{Q}_{l}$. Thus, we get from (1.7)

$$
V_{l}(T)^{*} \cong\left(Y(\bar{K}) \otimes_{\mathbb{Z}} \mathbb{Q}_{l}\right) \otimes_{\mathbb{Q}_{l}} V_{l}\left(\bar{K}^{\times}\right)^{*} .
$$

Let $\imath: \mathbb{Q}_{l} \hookrightarrow \mathbb{C}$ be a field embedding and let $F_{l, \imath}$ be the functor which associates to an $l$-adic representation of $\operatorname{Gal}(\bar{K} / K)$ a representation of $\mathcal{W}^{\prime}(\bar{K} / K)$. Clearly, the image of the representation of $\operatorname{Gal}(\bar{K} / K)$ on $Y(\bar{K}) \otimes_{\mathbb{Z}} \mathbb{Q}_{l}$ under $F_{l, \imath}$ is $\chi$ and the image of the representation of $\operatorname{Gal}(\bar{K} / K)$ on $V_{l}\left(\bar{K}^{\times}\right)^{*}$ under $F_{l, \imath}$ is $\omega^{-1}$. Since $F_{l, \imath}$ respects tensor products, by (1.8) the image $\lambda^{\prime}$ of the representation of $\operatorname{Gal}(\bar{K} / K)$ 
on $V_{l}(T)^{*}$ under $F_{l, \imath}$ is isomorphic to $\chi \otimes \omega^{-1}$. Thus, $\lambda^{\prime}$ is a representation of $\mathcal{W}(\bar{K} / K)$, i.e., $R=0$.

Lemma 1.12. Let $\rho^{\prime}=(\rho, P)$ be the representation of $\mathcal{W}^{\prime}(\bar{K} / K)$ associated to the natural $l$-adic representation of $\operatorname{Gal}(\bar{K} / K)$ on $V_{l}(G)^{*}$. Then $P=0$ and

$$
\rho \cong \kappa \oplus\left(\chi \otimes \omega^{-1}\right) \text {. }
$$

Proof. From the exact $\operatorname{Gal}(\bar{K} / K)$-equivariant sequence (1.2) we get the following exact sequence of $\operatorname{Gal}(\bar{K} / K)$-modules:

$$
0 \longrightarrow T(\bar{K}) \longrightarrow G(\bar{K}) \longrightarrow B(\bar{K}) \longrightarrow 0 .
$$

Since $T(\bar{K})$ is a divisible group, the last sequence induces an exact $\operatorname{Gal}(\bar{K} / K)$ equivariant sequence of $l$-adic Tate modules:

$$
0 \longrightarrow T_{l}(T) \longrightarrow T_{l}(G) \longrightarrow T_{l}(B) \longrightarrow 0 .
$$

By tensoring the above sequence with $\mathbb{Q}_{l}$ over $\mathbb{Z}_{l}$ and taking duals over $\mathbb{Q}_{l}$ afterwards, we get the following exact sequence of $\operatorname{Gal}(\bar{K} / K)$-modules:

$$
0 \longrightarrow V_{l}(B)^{*} \longrightarrow V_{l}(G)^{*} \longrightarrow V_{l}(T)^{*} \longrightarrow 0
$$

Sequence (1.9) induces an exact sequence of corresponding representations of $\mathcal{W}^{\prime}(\bar{K} / K)$, i.e.,

$$
0 \longrightarrow V_{l}(B)^{*} \otimes_{\imath} \mathbb{C} \stackrel{h}{\longrightarrow} V_{l}(G)^{*} \otimes_{\imath} \mathbb{C} \stackrel{g}{\longrightarrow} V_{l}(T)^{*} \otimes_{\imath} \mathbb{C} \longrightarrow 0
$$

is an exact sequence of $\mathcal{W}^{\prime}(\bar{K} / K)$-modules, where $\imath: \mathbb{Q}_{l} \hookrightarrow \mathbb{C}$ is a field embedding, $(\kappa, 0)$ is the representation of $\mathcal{W}^{\prime}(\bar{K} / K)$ on $V_{l}(B)^{*} \otimes_{\imath} \mathbb{C}, \rho^{\prime}=(\rho, P)$ is the representation of $\mathcal{W}^{\prime}(\bar{K} / K)$ on $V_{l}(G)^{*} \otimes_{\imath} \mathbb{C}$, and by Lemma 1.11 $\left(\chi \otimes \omega^{-1}, 0\right)$ is the representation of $\mathcal{W}^{\prime}(\bar{K} / K)$ on $V_{l}(T)^{*} \otimes_{\imath} \mathbb{C}$. In particular, (1.10) is an exact sequence of $\mathcal{W}(\bar{K} / K)$-modules and it splits if $\rho$ is semisimple, hence $\rho \cong \kappa \oplus\left(\chi \otimes \omega^{-1}\right)$. Thus, it is enough to show that $P=0$ and $\rho$ is semisimple.

Let $L \subset \bar{K}$ be a finite Galois extension of $K$ such that $T \times_{K} L$ splits and $B \times{ }_{K} L$ has good reduction. Since $\rho$ is semisimple if and only if its restriction to a subgroup of finite index is semisimple (Lemma 1.1) and $\operatorname{Res}_{\mathcal{W}^{\prime}(\bar{K} / L)}^{\mathcal{W}^{\prime}(\bar{K} / K)} \rho^{\prime}=\left(\operatorname{Res}_{K}^{L} \rho, P\right)([\underline{6}$, p. 130), to prove that $P=0$ and $\rho$ is semisimple we can assume that $T$ splits over $K$ and $B$ has good reduction over $K$. Then it follows from Lemma 1.11 that $\chi$ is trivial. Also, since the image of $I$ under $\rho$ is finite, by Lemma 1.1 to prove that $\rho$ is semisimple it is enough to prove that $\rho(\Phi)$ is diagonalizable.

Taking into account that $\chi$ is trivial, from (1.10) we obtain that in a suitable basis $\rho(\Phi)$ has the following form:

$$
\rho(\Phi)=\left(\begin{array}{cc}
\kappa(\Phi) & * \\
0 & q E_{r}
\end{array}\right)
$$

where $E_{r}$ is the $r \times r$-identity matrix. Let

$$
\psi_{l}: \operatorname{Gal}(\bar{K} / K) \longrightarrow \operatorname{Aut}\left(T_{l}(B)\right)
$$

be the $l$-adic representation corresponding to the Galois module $T_{l}(B)$. It is known that the absolute values of the eigenvalues of $\psi_{l}(\Phi)$ are equal to $q^{-1 / 2}$ ([9], Corollary on p. 499). Then the absolute values of the eigenvalues of $\kappa(\Phi)$ are equal to $q^{1 / 2}$, since the eigenvalues of $\kappa(\Phi)$ are the inverses of the eigenvalues of $\psi_{l}(\Phi)$. It follows that none of the eigenvalues of $\kappa(\Phi)$ is equal to $q$. Since $\kappa(\Phi)$ is diagonalizable by 
Lemma 1.2, formula (1.11) shows that $\rho(\Phi)$ is diagonalizable, hence $\rho$ is semisimple and $\rho^{\prime}$ is admissible.

Let us now show that $P=0$. Since (1.10) is an exact sequence of $\mathcal{W}^{\prime}(\bar{K} / K)$ modules, we have

$$
\begin{aligned}
P h(x) & =0, \quad \forall x \in V_{l}(B)^{*} \otimes_{\imath} \mathbb{C}, \\
g(P y) & =0, \quad \forall y \in V_{l}(G)^{*} \otimes_{\imath} \mathbb{C},
\end{aligned}
$$

which implies that $P^{2}=0$. On the other hand, since $\rho^{\prime}$ is admissible, it has the following form:

$$
\rho^{\prime} \cong \bigoplus_{i=1}^{s} \alpha_{i} \otimes \operatorname{sp}\left(n_{i}\right)
$$

where each $\alpha_{i}$ is a representation of $\mathcal{W}(\bar{K} / K)$, each $n_{i}$ is a positive integer, and we can assume that $n_{i} \neq n_{j}$ if $i \neq j$ ([6], p. 133, Cor. 2). Since $P^{2}=0$, it follows that each $n_{i}$ is 1 or 2, i.e., without loss of generality we can assume that

$$
\rho^{\prime} \cong \alpha_{1} \oplus\left(\alpha_{2} \otimes \operatorname{sp}(2)\right) .
$$

We will show that $\alpha_{2}=0$. Assume that $\alpha_{2} \neq 0$. From (1.12) we have

$$
\rho \cong \alpha_{1} \oplus \alpha_{2} \oplus\left(\alpha_{2} \otimes \omega\right) \text {. }
$$

On the other hand, since $\rho$ is semisimple, the exact sequence (1.10) of $\mathcal{W}(\bar{K} / K)$ modules splits, i.e.,

$$
\rho \cong \kappa \oplus\left(\omega^{-1}\right)^{\oplus r},
$$

because $\chi$ is trivial. Thus, combining the last two isomorphisms, we get

$$
\alpha_{1} \oplus \alpha_{2} \oplus\left(\alpha_{2} \otimes \omega\right) \cong \kappa \oplus\left(\omega^{-1}\right)^{\oplus r} .
$$

By assumption, $B$ has good reduction, hence by the criterion of Néron-Ogg-Shafarevič (9], p. 493, Thm. 1) the inertia group $I$ acts trivially on $V_{l}(B)^{*}$. Since $\kappa$ is semisimple by Lemma 1.2. this implies that $\kappa \cong \bigoplus_{i=1}^{2 m} \kappa_{i}$, where $m=\operatorname{dim} B$ and $\kappa_{1}, \ldots, \kappa_{2 m}$ are one-dimensional representations of $\mathcal{W}(\bar{K} / K)$. Thus, it follows from (1.13) that $\alpha_{2}$ is isomorphic to a sum of one-dimensional representations. Let $\alpha_{0}$ be one of them. Then using the uniqueness of decomposition of a semisimple module into simple modules we have from (1.13):

$$
\alpha_{0} \cong \omega^{-1} \quad \text { or } \quad \alpha_{0} \cong \kappa_{i}
$$

for some $\kappa_{i}$, hence

$$
\alpha_{0} \otimes \omega \cong 1 \quad \text { or } \quad \alpha_{0} \otimes \omega \cong \kappa_{i} \otimes \omega .
$$

In particular, the absolute value of $\alpha_{0} \otimes \omega(\Phi)$ is 1 or $q^{-1 / 2}$, because the absolute value of $\kappa_{i}(\Phi)$ is $q^{1 / 2}$ for each $i$ (see above). This implies that $\alpha_{0} \otimes \omega$ is neither $\omega^{-1}$ nor $\kappa_{j}$ for any $j$ which contradicts (1.13). Thus, $\alpha_{2}=0$ and $\rho^{\prime}$ is a representation of $\mathcal{W}(\bar{K} / K)$.

Lemma 1.13. $\sigma^{\prime}$ is admissible.

Proof. There is the following exact $\operatorname{Gal}(\bar{K} / K)$-equivariant sequence (4, p. 312):

$$
0 \longrightarrow G(\bar{K})_{l^{n}} \longrightarrow A(\bar{K})_{l^{n}} \longrightarrow Y(\bar{K}) / l^{n} Y(\bar{K}) \longrightarrow 0,
$$


where $G(\bar{K})_{l^{n}}$ (respectively $A(\bar{K})_{l^{n}}$ ) denotes $\operatorname{Hom}\left(\mathbb{Z} / l^{n} \mathbb{Z}, G(\bar{K})\right.$ ) (respectively $\operatorname{Hom}\left(\mathbb{Z} / l^{n} \mathbb{Z}, A(\bar{K})\right)$. Since $Y(\bar{K})$ is a free group of rank $r$ and $G(\bar{K})$ is divisible by Lemma B.1 (see Appendix B), we have the following exact sequence of $\operatorname{Gal}(\bar{K} / K)$-modules:

$$
0 \longrightarrow T_{l}(G) \longrightarrow T_{l}(A) \longrightarrow \chi \otimes \mathbb{Z}_{l} \longrightarrow 0 .
$$

By tensoring the above sequence with $\mathbb{Q}_{l}$ over $\mathbb{Z}_{l}$ and taking duals over $\mathbb{Q}_{l}$ afterwards, we get:

$$
0 \longrightarrow \chi \otimes \mathbb{Q}_{l} \longrightarrow V_{l}(A)^{*} \longrightarrow V_{l}(G)^{*} \longrightarrow 0,
$$

because $\chi \otimes \mathbb{Q}_{l} \cong\left(\chi \otimes \mathbb{Q}_{l}\right)^{*}$.

As in the proof of Lemma 1.12 by Lemma 1.1 we can assume that $B$ has good reduction over $K$ and $T$ splits over $K$. Also, by Lemma 1.1 to prove that $\sigma^{\prime}$ is admissible it is enough to prove that $\sigma(\Phi)$ is diagonalizable.

Sequence (1.14) induces an exact sequence of corresponding representations of $\mathcal{W}^{\prime}(\bar{K} / K)$, i.e.,

$$
0 \longrightarrow\left(\chi \otimes \mathbb{Q}_{l}\right) \otimes_{\imath} \mathbb{C} \longrightarrow V_{l}(A)^{*} \otimes_{\imath} \mathbb{C} \longrightarrow V_{l}(G)^{*} \otimes_{\imath} \mathbb{C} \longrightarrow 0
$$

is an exact sequence of $\mathcal{W}^{\prime}(\bar{K} / K)$-modules, where $\chi$ is the representation of $\mathcal{W}^{\prime}(\bar{K} / K)$ on $\left(\chi \otimes \mathbb{Q}_{l}\right) \otimes_{\imath} \mathbb{C}, \sigma^{\prime}=(\sigma, N)$ is the representation of $\mathcal{W}^{\prime}(\bar{K} / K)$ on $V_{l}(A)^{*} \otimes_{\imath} \mathbb{C}$, and by Lemma 1.12, $\kappa \oplus\left(\chi \otimes \omega^{-1}\right)$ is the representation of $\mathcal{W}^{\prime}(\bar{K} / K)$ on $V_{l}(G)^{*} \otimes_{l} \mathbb{C}$. Taking into account that $\chi$ is trivial and (1.15) is an exact sequence of $\mathcal{W}(\bar{K} / K)$-modules, we obtain that in a suitable basis $\sigma(\Phi)$ has the following form:

$$
\sigma(\Phi)=\left(\begin{array}{ccc}
E_{r} & * & * \\
0 & q E_{r} & * \\
0 & 0 & \kappa(\Phi)
\end{array}\right) .
$$

Here $\kappa(\Phi)$ is diagonalizable by Lemma 1.2, Since the absolute values of the eigenvalues of $\kappa(\Phi)$ are equal to $q^{1 / 2}$ (see above), none of the eigenvalues of $\kappa(\Phi)$ is equal to 1 or $q$. Thus, (1.16) shows that $\sigma(\Phi)$ is diagonalizable, hence $\sigma$ is semisimple, and $\sigma^{\prime}$ is admissible.

Proof of Proposition 1.10. Since $\sigma^{\prime}$ is admissible by Lemma 1.13 and the representations of the Weil-Deligne group $\mathcal{W}^{\prime}(\bar{K} / K)$ on $\left(\chi \otimes \mathbb{Q}_{l}\right) \otimes_{\imath} \mathbb{C}$ and $V_{l}(G)^{*} \otimes_{\imath} \mathbb{C}$ are actually representations of the Weil group $\mathcal{W}(\bar{K} / K)$, the same argument as in the proof of Lemma 1.12 applied to (1.15) gives that $\sigma^{\prime}$ has the following form:

$$
\sigma^{\prime} \cong \gamma \oplus(\delta \otimes \operatorname{sp}(2)),
$$

where $\gamma$ and $\delta$ are representations of $\mathcal{W}(\bar{K} / K)$. Hence

$$
\sigma \cong \gamma \oplus \delta \oplus(\delta \otimes \omega) \text {. }
$$

On the other hand, since $\sigma$ is semisimple by Lemma 1.13, the exact sequence (1.15) of $\mathcal{W}(\bar{K} / K)$-modules splits, i.e.,

$$
\sigma \cong \chi \oplus \kappa \oplus\left(\chi \otimes \omega^{-1}\right) .
$$

Thus, combining the last two isomorphisms, we get

$$
\gamma \oplus \delta \oplus(\delta \otimes \omega) \cong \chi \oplus \kappa \oplus\left(\chi \otimes \omega^{-1}\right) .
$$

Note that $\chi$ is isomorphic to a subrepresentation of $\gamma \oplus(\delta \otimes \omega)$, because by (1.15)

$$
\chi \hookrightarrow \operatorname{ker} N \cong \gamma \oplus(\delta \otimes \omega) .
$$


Thus, $\delta$ is isomorphic to a subrepresentation of $\kappa \oplus\left(\chi \otimes \omega^{-1}\right)$ by the uniqueness of decomposition of a semisimple module into simple modules. We claim that $\delta$ is isomorphic to a subrepresentation of $\chi \otimes \omega^{-1}$. Indeed, suppose there is an irreducible subrepresentation $\delta_{0}$ of $\delta$ which is isomorphic to a subrepresentation of $\kappa$. Since the absolute values of the eigenvalues of $\kappa(\Phi)$ are equal to $q^{1 / 2}$ (see above), the eigenvalues of $\delta_{0}(\Phi)$ are of absolute value $q^{1 / 2}$. Hence the eigenvalues of $\delta_{0} \otimes \omega(\Phi)$ are of absolute value $q^{-1 / 2}$. On the other hand, it follows from (1.18) that $\delta_{0} \otimes \omega$ is isomorphic to a subrepresentation of $\chi, \kappa$, or $\chi \otimes \omega^{-1}$, which is a contradiction because the eigenvalues of $\chi(\Phi), \kappa(\Phi)$, and $\chi \otimes \omega^{-1}(\Phi)$ are of absolute values 1 , $q^{1 / 2}$, and $q$ respectively. Thus, $\delta$ is isomorphic to a subrepresentation of $\chi \otimes \omega^{-1}$. Since $\operatorname{dim} \delta=r$ by Lemma B.2 (see Appendix [B), we have $\delta \cong \chi \otimes \omega^{-1}$, hence $\gamma \cong \kappa$ by (1.18) and $\sigma^{\prime} \cong \kappa \oplus\left(\chi \otimes \omega^{-1} \otimes \operatorname{sp}(2)\right)$ by (1.17).

Remark 1.14. To prove that $\sigma^{\prime} \cong \gamma \oplus(\delta \otimes \mathrm{sp}(2))$ for some representations $\gamma$ and $\delta$ of $\mathcal{W}(\bar{K} / K)$ it is enough to use the theorem on semistable reduction. However, to find a formula for $W\left(\sigma^{\prime} \otimes \tau\right)$ (see Corollary 1.16 below) it is essential that $\gamma$ comes from an abelian variety over $K$ with potential good reduction and $\delta$ comes from a torus over $K$.

Corollary 1.15. The representation $\sigma^{\prime}$ does not depend on the choice of $l$ and $\imath$.

Proof. The statement is a consequence of Corollary 1.4 and Proposition 1.10

Corollary 1.16. Let $\tau$ be a representation of $\mathrm{Gal}(\bar{K} / K)$ with real-valued character. Then

$$
W\left(\sigma^{\prime} \otimes \tau\right)=W(\kappa \otimes \tau) \cdot \operatorname{det} \tau(-1)^{r} \cdot \operatorname{det} \chi(-1)^{\operatorname{dim} \tau} \cdot(-1)^{\langle\chi, \tau\rangle} .
$$

Moreover, when $p>2 g+1$ we have

$$
W\left(\sigma^{\prime} \otimes \tau\right)=\operatorname{det} \mu(-1)^{\operatorname{dim} \tau} \cdot \operatorname{det} \chi(-1)^{\operatorname{dim} \tau} \cdot \operatorname{det} \tau(-1)^{r+l_{1}} \cdot \alpha^{\operatorname{dim} \tau} \cdot(-1)^{\langle\chi, \tau\rangle+l_{2}},
$$

where $l_{1}=\operatorname{dim} \mu+\frac{1}{2}\left(\operatorname{dim} \mu_{1}+\cdots+\operatorname{dim} \mu_{a}\right), \mu$ is a representation of $\mathcal{W}(\bar{K} / K)$, $\mu_{1}, \ldots, \mu_{a}$ are irreducible symplectic subrepresentations of $\kappa \otimes \omega^{1 / 2}, \alpha= \pm 1, l_{2}=$ $a \cdot\langle 1, \tau\rangle+a \cdot\langle\eta, \tau\rangle+\left\langle\hat{\mu}_{1} \oplus \cdots \oplus \hat{\mu}_{a}, \tau\right\rangle, \hat{\mu}_{1}, \ldots, \hat{\mu}_{a}$ are representations given by (1.4) such that $\hat{\mu}_{1} \oplus \cdots \oplus \hat{\mu}_{a}$ is realizable over $\mathbb{Q}$, and $\eta$ is the unramified quadratic character of $K^{\times}$.

Proof. Since the root number of a direct sum of representations of $\mathcal{W}^{\prime}(\bar{K} / K)$ equals the product of the root numbers of the summands, we get from Proposition 1.10

$$
W\left(\sigma^{\prime} \otimes \tau\right)=W(\kappa \otimes \tau) \cdot W\left(\chi \otimes \omega^{-1} \otimes \operatorname{sp}(2) \otimes \tau\right)
$$

where by Proposition 6 on p. 327 of [7]

$$
W\left(\chi \otimes \omega^{-1} \otimes \operatorname{sp}(2) \otimes \tau\right)=\operatorname{det} \tau(-1)^{r} \cdot \operatorname{det} \chi(-1)^{\operatorname{dim} \tau} \cdot(-1)^{\langle\chi, \tau\rangle},
$$

which proves (1.19).

Formula (1.20) is a consequence of (1.19) together with Proposition 1.9. 


\section{Proof of Theorem 0.2}

We keep the notation of the introduction.

Lemma 2.1. Let $A$ be an abelian variety of dimension $g$ over a number field $F$ and $\tau$ a representation of $\operatorname{Gal}(\bar{F} / F)$ with real-valued character. Then at every infinite place $v$ of $F$ we have

$$
W\left(A_{v}, \tau_{v}\right)=(-1)^{g \operatorname{dim} \tau} .
$$

Proof. To define $W\left(A_{v}, \tau_{v}\right)$ let $\sigma_{v}^{\prime}$ denote the representation of the Weil-Deligne group $\mathcal{W}^{\prime}\left(\bar{F}_{v} / F_{v}\right)$ associated to the components of $H^{1}\left(A_{v}(\mathbb{C}), \mathbb{C}\right)$ in the Hodge decomposition; then $W\left(A_{v}, \tau_{v}\right)=W\left(\sigma_{v}^{\prime} \otimes \tau_{v}\right)$, where $\tau_{v}$ is viewed as a representation of $\mathcal{W}^{\prime}\left(\bar{F}_{v} / F_{v}\right)$. If $v$ is an infinite place such that $F_{v} \cong \mathbb{C}$, then the representation $\sigma_{v}^{\prime}=\sigma_{v}$ of $\mathcal{W}^{\prime}(\mathbb{C} / \mathbb{C})=\mathcal{W}(\mathbb{C} / \mathbb{C})=\mathbb{C}^{\times}$has the following form:

$$
\sigma_{v}=\left(\varphi_{1,0} \otimes H^{1,0}\right) \oplus\left(\varphi_{0,1} \otimes H^{0,1}\right),
$$

where $\varphi_{p, q}: \mathcal{W}(\mathbb{C} / \mathbb{C}) \longrightarrow \mathbb{C}^{\times}(p, q \in \mathbb{Z})$ are given by

$$
\varphi_{p, q}(z)=z^{-p} \bar{z}^{-q}
$$

and $H^{1,0}$ and $H^{0,1}$ are the components of $H^{1}\left(A_{v}(\mathbb{C}), \mathbb{C}\right)$ in the Hodge decomposition:

$$
H^{1}\left(A_{v}(\mathbb{C}), \mathbb{C}\right)=H^{1,0} \oplus H^{0,1}
$$

Here $H^{1,0}$ and $H^{0,1}$ are endowed with the trivial action of $\mathcal{W}(\mathbb{C} / \mathbb{C})$, hence

$$
\sigma_{v}=\left(\varphi_{1,0} \oplus \varphi_{0,1}\right)^{\oplus g}
$$

Let $v$ be an infinite place such that $F_{v} \cong \mathbb{R}$. We have

$$
\mathcal{W}^{\prime}(\mathbb{C} / \mathbb{R})=\mathcal{W}(\mathbb{C} / \mathbb{R})=\mathbb{C}^{\times} \cup J \mathbb{C}^{\times},
$$

where $J^{2}=-1$ and $J z J^{-1}=\bar{z}$ for $z \in \mathbb{C}^{\times}$. Here $\mathcal{W}(\mathbb{C} / \mathbb{C})$ is identified with the subgroup $\mathbb{C}^{\times}$of $\mathcal{W}(\mathbb{C} / \mathbb{R})$. In this case the representation $\sigma_{v}^{\prime}=\sigma_{v}$ of $\mathcal{W}(\mathbb{C} / \mathbb{R})$ has the following form:

$$
\sigma_{v}=\operatorname{Ind}_{\mathbb{R}}^{\mathbb{C}} \varphi_{0,1} \otimes H^{0,1},
$$

where $\operatorname{Ind}_{\mathbb{R}}^{\mathbb{C}} \varphi_{0,1}$ denotes the representation of $\mathcal{W}(\mathbb{C} / \mathbb{R})$ induced from $\varphi_{0,1}$. As in the complex case, $H^{0,1}$ is endowed with the trivial action of $\mathcal{W}(\mathbb{C} / \mathbb{R})$, hence

$$
\sigma_{v}=\left(\operatorname{Ind}_{\mathbb{R}}^{\mathbb{C}} \varphi_{0,1}\right)^{\oplus g}
$$

([6], p. $155, \S 20)$.

It follows from the proof of Theorem 2(i) on p. 329 of [7] that

$$
\begin{aligned}
W\left(\left(\varphi_{1,0} \oplus \varphi_{0,1}\right) \otimes \tau_{v}\right) & =(-1)^{\operatorname{dim} \tau} \quad \text { if } \quad F_{v} \cong \mathbb{C} \text { and } \\
W\left(\left(\operatorname{Ind}_{\mathbb{R}}^{\mathbb{C}} \varphi_{0,1}\right) \otimes \tau_{v}\right) & =(-1)^{\operatorname{dim} \tau} \text { if } \quad F_{v} \cong \mathbb{R} .
\end{aligned}
$$

Now the statement follows from these formulas together with formulas (2.1) and (2.2).

Lemma 2.2. Let $G$ be a finite group, $D \subseteq G$ an abelian subgroup, and $\tau$ an irreducible representation of $G$ with real-valued character. If $m_{\mathbb{Q}}(\tau)=2$, then $\operatorname{Res}_{D}^{G} \tau$ is symplectic ([7], Lemma on p. 347).

Lemma 2.3. Let $G$ be a finite group and $\tau$ an irreducible representation of $G$ with real-valued character. If $m_{\mathbb{Q}}(\tau)=2$ then $\operatorname{dim} \tau$ is even and $\operatorname{det} \tau$ is trivial. 
Proof. By the Lemma on p. 339 of [7] if $\tau$ has odd dimension or a nontrivial determinant, then there is a cyclic subgroup $D$ of $G$ such that $\operatorname{Res}_{D}^{G} \tau$ is not symplectic, which contradicts Lemma 2.2 .

Proof of Theorem 0.2 , By Lemmas 2.1 and 2.3, $W\left(A_{v}, \tau_{v}\right)=1$ at every infinite place $v$ of $F$.

Let $v$ be a finite place of $F$ lying over a prime number $p$. Let $\sigma_{v}^{\prime}$ be the representation of $\mathcal{W}^{\prime}\left(\bar{F}_{v} / F_{v}\right)$ associated to the first cohomology of $A_{v}$. Since by Lemma $2.3 \operatorname{det} \tau$ is trivial and $\operatorname{dim} \tau$ is even, (1.19) implies

$$
W\left(A_{v}, \tau_{v}\right)=W\left(\kappa_{v} \otimes \tau_{v}\right) \cdot(-1)^{\left\langle\chi_{v}, \tau_{v}\right\rangle},
$$

where $\chi_{v}$ is a representation of $\operatorname{Gal}\left(\bar{F}_{v} / F_{v}\right)$ realizable over $\mathbb{Z}$ (see Subsection 1.3 for the definition of $\chi_{v}$ ). Moreover, when $p>2 g+1$ from (1.20) we have

$$
W\left(A_{v}, \tau_{v}\right)=(-1)^{a\left\langle 1, \tau_{v}\right\rangle+a\left\langle\eta_{v}, \tau_{v}\right\rangle+\left\langle\lambda_{v}, \tau_{v}\right\rangle+\left\langle\chi_{v}, \tau_{v}\right\rangle},
$$

where $a \in \mathbb{N}, \eta_{v}$ is the unramified quadratic character of $F_{v}^{\times}$, and $\lambda_{v}$ is a representation of $\operatorname{Gal}\left(\bar{F}_{v} / F_{v}\right)$ realizable over $\mathbb{Q}$.

The rest of the proof is analogous to the argument given by D. Rohrlich in [7]. Let $K \subset \bar{F}$ be a finite Galois extension of $F$ such that $\tau$ factors through the group $G=\operatorname{Gal}(K / F)$ and $\chi_{v}$ factors through the decomposition subgroup $H$ of $G$ at $v$. Then

$$
\left\langle\chi_{v}, \tau_{v}\right\rangle=\left\langle\operatorname{Ind}_{H}^{G} \chi_{v}, \tau\right\rangle
$$

by Frobenius reciprocity. Since $\chi_{v}$ is realizable over $\mathbb{Q}, \operatorname{Ind}_{H}^{G} \chi_{v}$ is realizable over $\mathbb{Q}$, hence $\left\langle\operatorname{Ind}_{H}^{G} \chi_{v}, \tau\right\rangle$ is divisible by $m_{\mathbb{Q}}(\tau)$. By assumption $m_{\mathbb{Q}}(\tau)=2$, hence $\left\langle\chi_{v}, \tau_{v}\right\rangle$ is even. Analogously, $\left\langle 1, \tau_{v}\right\rangle,\left\langle\eta_{v}, \tau_{v}\right\rangle$, and $\left\langle\lambda_{v}, \tau_{v}\right\rangle$ are even, hence $W\left(A_{v}, \tau_{v}\right)=$ $W\left(\kappa_{v} \otimes \tau_{v}\right)$ by (2.3), and when $p>2 g+1$ we have $W\left(A_{v}, \tau_{v}\right)=1$ by (2.4). When $p \leq 2 g+1$ the decomposition subgroup of $\operatorname{Gal}(L / F)$ at $v$ is abelian by assumption, hence $\tau_{v}$ is symplectic by Lemma 2.2. Also, $\kappa_{v} \otimes \omega_{v}{ }^{1 / 2}$ is symplectic, because $\kappa_{v}$ comes from an abelian variety (see Subsection 1.1). Since $\kappa_{v}$ is a representation of $\mathcal{W}\left(\bar{F}_{v} / F_{v}\right)$ (see Subsection [1.2) and real powers of $\omega_{v}$ do not change the root number,

$$
W\left(\kappa_{v} \otimes \tau_{v}\right)=W\left(\kappa_{v} \otimes \omega_{v}^{1 / 2} \otimes \tau_{v}\right)=1
$$

by Proposition 2 and the remark after it on p. 319 of 7 .

Remark 2.4. If $\tau$ is symplectic, then Theorem 0.2 follows from the fact that the root number of the tensor product of two symplectic admissible representations of the Weil-Deligne group of a local non-Archimedean field equals 1 . Note that if $\tau$ is symplectic, then $m_{\mathbb{Q}}(\tau)=2$ but not vice versa: there are examples of irreducible orthogonal representations of finite groups with the Schur index over the rationals equal to 2 .

\section{Appendix A.}

Lemma A.1. Let $C=\langle c\rangle$ be an infinite cyclic group generated by an element $c$ and let $E=\langle e\rangle$ be a finite cyclic group of order $n$ generated by an element e. Let $G=E \rtimes C$ be a semi-direct product, where $C$ acts on $E$ via $c^{-1} e c=e^{k}$ for some $k \in(\mathbb{Z} / n \mathbb{Z})^{\times}$. Denote by $s$ the order of $k$ in $(\mathbb{Z} / n \mathbb{Z})^{\times}$. Then every irreducible representation $\lambda$ of $G$ has the following form:

$$
\lambda=\lambda_{0} \otimes \phi,
$$


where $\lambda_{0}$ is an irreducible representation of $G$ trivial on the subgroup of $C$ generated by $c^{s}$ and $\phi$ is a one-dimensional representation of $G$.

Proof. Since $c^{s}$ is contained in the center of $G$ and $\lambda$ is an irreducible complex representation, by Schur's lemma $\lambda\left(c^{s}\right)$ acts as a scalar $a \in \mathbb{C}^{\times}$. Define a onedimensional representation $\phi$ of $G$ as follows: $\phi(e)=1$ and $\phi(c)$ equals an $s$-th root of $a$. Then $\lambda_{0}=\lambda \otimes \phi^{-1}$ is trivial on $\left\langle c^{s}\right\rangle$ and $\lambda=\lambda_{0} \otimes \phi$.

Proof of Proposition 1.5. Let $\lambda$ be an irreducible symplectic representation of $G$. Then by Lemma A.1 $\lambda=\lambda_{0} \otimes \phi$, where $\lambda_{0}$ is an irreducible representation of $G$ trivial on the subgroup of $C$ generated by $c^{s}$ and $\phi$ is a one-dimensional representation of $G$. Since $\lambda$ is symplectic, $\lambda$ and its contragredient representation have the same character, which implies that for any $g \in G$ we have

$$
\phi(g) \cdot \operatorname{tr} \lambda_{0}(g)=\phi(g)^{-1} \cdot \operatorname{tr} \lambda_{0}\left(g^{-1}\right) .
$$

Taking into account that $\lambda_{0}$ is trivial on $\left\langle c^{s}\right\rangle$, the above equation for $g=c^{s}$ gives $\phi\left(c^{2 s}\right)=1$, i.e., $\lambda$ can be considered as an irreducible symplectic representation of the finite group $H=G /\left\langle c^{2 s}\right\rangle \cong E \rtimes C /\left\langle c^{2 s}\right\rangle$. By abuse of notation we will denote the image of $c$ in $C /\left\langle c^{2 s}\right\rangle$ also by $c$, then $c^{2 s}=1$ and $c^{-1} e c=e^{k}$ in $H$. As an irreducible representation of the semi-direct product $H, \lambda$ can be constructed from a one-dimensional representation $\psi_{1}$ of $E$ in the following way. Let $\psi_{1}(e)=\xi$ for some $n$-th root of unity $\xi$ of order $d$ in $\mathbb{C}^{\times}$. Let $\Gamma=\left\langle c^{x}\right\rangle$, where $x=|k|$ in $(\mathbb{Z} / d \mathbb{Z})^{\times}$, and $\psi_{2}$ be a one-dimensional representation of $\Gamma$. Then $\psi_{1}$ and $\psi_{2}$ can be extended to representations of $E \rtimes \Gamma$ via

$$
\begin{aligned}
& \psi_{1}\left(c^{x v} e^{t}\right)=\psi_{1}\left(e^{t}\right), \\
& \psi_{2}\left(c^{x v} e^{t}\right)=\psi_{2}\left(c^{x v}\right) .
\end{aligned}
$$

Then $\lambda=\operatorname{Ind}_{E \rtimes \Gamma}^{H}\left(\psi_{1} \otimes \psi_{2}\right)$ ([8], p. 62, Prop. 25). Let $W$ be a representation space of $H$ corresponding to $\lambda$, and $V=\mathbb{C} b \subseteq W$ be a one-dimensional subrepresentation of $\operatorname{Res}_{E \rtimes \Gamma}^{H} \lambda$ isomorphic to $\psi_{1} \otimes \psi_{2}$ and spanned by a nonzero vector $b \in V$ over $\mathbb{C}$. Then $W=V \oplus c V \oplus c^{2} V \oplus \cdots \oplus c^{x-1} V$, and $\lambda$ has the following form in the basis $\left\{b, c b, c^{2} b, \ldots, c^{x-1} b\right\}$ :

$$
\lambda(e)=\left(\begin{array}{ccccc}
\xi & 0 & 0 & \ldots & 0 \\
0 & \xi^{k} & 0 & \ldots & 0 \\
0 & 0 & \xi^{k^{2}} & \ldots & 0 \\
\vdots & \vdots & \vdots & \ddots & \vdots \\
0 & 0 & 0 & \ldots & \xi^{k^{x-1}}
\end{array}\right), \quad \lambda(c)=\left(\begin{array}{ccccc}
0 & 0 & 0 & \ldots & \psi_{2}\left(c^{x}\right) \\
1 & 0 & 0 & \ldots & 0 \\
0 & 1 & 0 & \ldots & 0 \\
\vdots & \vdots & \vdots & \ddots & \vdots \\
0 & 0 & 0 & \ldots & 0
\end{array}\right) .
$$

Since $\lambda$ is symplectic, $x=\operatorname{dim} \lambda$ is even and $\operatorname{det} \lambda=1$, hence $\operatorname{det} \lambda(c)=-\psi_{2}\left(c^{x}\right)=$ 1 , which implies that $\psi_{2}\left(c^{x}\right)=-1$. Denote by $\chi$ the character of $\lambda$. By Proposition 39 ([8, p. 109), $\lambda$ is symplectic if and only if

$$
-1=\frac{1}{|H|} \cdot \sum_{y \in H} \chi\left(y^{2}\right) .
$$

Let $y=c^{v} e^{t}$, consequently, $y^{2}=c^{2 v} e^{t\left(1+k^{v}\right)}$. Clearly, $\chi\left(y^{2}\right)=0$ if $y^{2} \notin E \rtimes \Gamma$ and $y^{2} \in E \rtimes \Gamma$ if and only if $x$ divides $2 v$ and, since $x$ is even, if and only if $\frac{x}{2}$ divides 
$v$. Let $v=\frac{x}{2} m$; then we have

$$
\begin{aligned}
\sum_{y \in H} \chi\left(y^{2}\right) & =\sum_{\substack{y \in H \\
y^{2} \in E \rtimes \Gamma}} \chi\left(y^{2}\right)=\sum_{t, m} \chi\left(c^{m x} e^{t\left(1+k^{v}\right)}\right) \\
= & \sum_{\substack{m, t \\
m \text { even }}} \chi\left(e^{t\left(1+k^{v}\right)}\right)-\sum_{\substack{m, t \\
m \text { odd }}} \chi\left(e^{t\left(1+k^{v}\right)}\right) .
\end{aligned}
$$

Let $S_{1}=\sum_{m \text { even }} \sum_{t} \chi\left(e^{t\left(1+k^{v}\right)}\right)$ and $S_{2}=\sum_{m \text { odd }} \sum_{t} \chi\left(e^{t\left(1+k^{v}\right)}\right)$.

If $m$ is even, then $v=x\left(\frac{m}{2}\right)$ and, since $x=|k|$ in $(\mathbb{Z} / d \mathbb{Z})^{\times}, 1+k^{v} \equiv 2(\bmod d)$. Since $\chi\left(e^{t}\right)=\xi^{t}+\xi^{k t}+\cdots+\xi^{k^{x-1} t}$ and $\xi^{d}=1$, we have $\chi\left(e^{t\left(1+k^{v}\right)}\right)=\chi\left(e^{2 t}\right)$ and $S_{1}=\sum_{m \text { even }} \sum_{t} \chi\left(e^{2 t}\right)$. We will show that $\sum_{t} \chi\left(e^{2 t}\right)=0$. First, note that if $d=1,2$, then $\lambda$ is one-dimensional, hence cannot be symplectic.

If $r \in \mathbb{Z}$ and $r \equiv 0(\bmod d)$, then

$$
\sum_{t=0}^{n-1} \chi\left(e^{r t}\right)=\sum_{t=0}^{n-1} \sum_{j=0}^{x-1} \xi^{r t k^{j}}=n x .
$$

If $r \in \mathbb{Z}$ and $r \not \equiv 0(\bmod d)$, then

$$
\sum_{t=0}^{n-1} \chi\left(e^{r t}\right)=\sum_{t=0}^{n-1} \sum_{j=0}^{x-1} \xi^{r t k^{j}}=\sum_{j=0}^{x-1} \frac{1-\xi^{r n k^{j}}}{1-\xi^{r k^{j}}}=0 .
$$

Thus

$$
\sum_{t=0}^{n-1} \chi\left(e^{r t}\right)=\left\{\begin{array}{cl}
n x, & r \equiv 0(\bmod d) \\
0, & r \neq \equiv 0(\bmod d) .
\end{array}\right.
$$

Since $d \neq 1,2$, formula A.3 implies that $S_{1}=0$.

If $m$ is odd, then $k^{v} \equiv k^{\frac{x}{2}}(\bmod d)$, hence $\chi\left(e^{t\left(1+k^{v}\right)}\right)=\chi\left(e^{t\left(1+k^{\frac{x}{2}}\right)}\right)$. Thus

$$
S_{2}=\sum_{m \text { odd }} \sum_{t=0}^{n-1} \chi\left(e^{t\left(1+k^{v}\right)}\right)=\frac{2 s}{x} \cdot \sum_{t=0}^{n-1} \chi\left(e^{t\left(1+k^{\frac{x}{2}}\right)}\right)
$$

and by (A.3) we have

$$
S_{2}=\left\{\begin{array}{cl}
2 s n, & 1+k^{\frac{x}{2}} \equiv 0(\bmod d) \\
0, & 1+k^{\frac{x}{2}} \neq \equiv(\bmod d) .
\end{array}\right.
$$

Hence by (A.2)

$$
\frac{1}{|H|} \cdot \sum_{y \in H} \chi\left(y^{2}\right)=\frac{1}{2 s n} \cdot\left(S_{1}-S_{2}\right)=-\frac{S_{2}}{2 s n},
$$

which together with (A.1) and (A.4) proves the proposition.

If $U$ is a complex finite-dimensional vector space and $\lambda: D \longrightarrow \mathrm{GL}(U)$ is a representation of a group $D$ on $U$, then by $\check{\lambda}: D \longrightarrow \operatorname{GL}(\check{U})$ we denote the representation of $D$ on $\check{U}$, where $\check{U}$ is a $\mathbb{C}[D]$-module with the underlying $D$-module $U^{*}$ and multiplication by constants defined as follows:

$$
a \cdot \phi=\bar{a} \phi, \quad a \in \mathbb{C}, \phi \in U^{*} .
$$


We say that $U$ is unitary if $U$ admits a nondegenerate invariant hermitian form (not necessarily positive definite).

Lemma A.2. Every semisimple unitary, orthogonal, or symplectic representation $\lambda$ of a group $D$ has the form

$$
\lambda \cong \nu \oplus \tilde{\nu} \oplus \lambda_{1}^{z_{1}} \oplus \cdots \oplus \lambda_{t}^{z_{t}},
$$

where $\nu$ is a representation of $D, \tilde{\nu}=\nu^{*}$ if $\lambda$ is orthogonal or symplectic and $\tilde{\nu}=\check{\nu}$ if $\lambda$ is unitary, and $\lambda_{1}, \ldots, \lambda_{t}$ are pairwise nonisomorphic irreducible unitary, orthogonal, or symplectic representations of $D$ respectively.

Proof of Lemma A.2. We say that a unitary, orthogonal, or symplectic representation is minimal if it cannot be written as an orthogonal sum of nonzero invariant subspaces. Clearly, every unitary, orthogonal, or symplectic representation is an orthogonal sum of minimal unitary, orthogonal, or symplectic representations respectively. Thus, it is enough to prove that if $\lambda$ is a semisimple minimal unitary, orthogonal, or symplectic representation of $D$, then either $\lambda$ is irreducible or $\lambda \cong \nu \oplus \tilde{\nu}$ for some irreducible representation $\nu$ of $D$. Let $U$ be a representation space of $D$ corresponding to $\lambda, \tilde{U}=U^{*}$ if $\lambda$ is orthogonal or symplectic and $\tilde{U}=\check{U}$ if $\lambda$ is unitary. Since $\lambda$ is semisimple, $U=V_{1} \oplus \cdots \oplus V_{n}$, where $V_{1}, \ldots, V_{n}$ are nonzero simple $\mathbb{C}[D]$-submodules of $U$. Let $\langle\cdot, \cdot\rangle$ be a nondegenerate invariant form on $U$. It defines a $\mathbb{C}[D]$-module isomorphism $\phi$ between $U$ and $\tilde{U}$ via $\phi(u)=\langle u, \cdot\rangle$, $u \in U$. Let $\psi: \tilde{U} \longrightarrow \tilde{V}_{1} \oplus \cdots \oplus \tilde{V}_{n}$ denote the usual isomorphism between $\tilde{U}=\left(V_{1} \oplus \cdots \oplus V_{n}\right)^{\sim}$ and $\tilde{V}_{1} \oplus \cdots \oplus \tilde{V}_{n}$. For each $i$ and $j$ let $\alpha_{i j}: V_{i} \longrightarrow \tilde{V}_{j}$ be a $\mathbb{C}[D]$-module homomorphism defined by the following diagram:

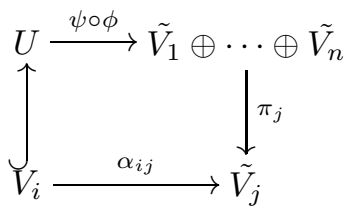

where $\pi_{j}$ is the projection onto the $j$-th factor. Since $\psi \circ \phi$ is an isomorphism, there exists some $\tilde{V}_{i}$ such that $\alpha_{1 i} \neq 0$, which implies that $\alpha_{1 i}$ is an isomorphism, since $V_{1}, \ldots, V_{n}$ are simple. If $i=1$, then it follows that $\left.\langle\cdot, \cdot\rangle\right|_{V_{1}}$ is nondegenerate, hence $V_{1}$ and its orthogonal complement are invariant subspaces of $U$. Since $U$ is minimal, this implies that $U=V_{1}$ and $U$ is irreducible. Thus, we can assume that for each $j$ we have $\alpha_{j j}=0$, which is equivalent to $\left\langle V_{j}, V_{j}\right\rangle=0$. Without loss of generality we can assume that $\alpha_{12} \neq 0$. Then $\alpha_{21} \neq 0$. Indeed, if $\alpha_{12} \neq 0$, then there is some $u \in V_{1}$ such that $\left.\langle u, \cdot\rangle\right|_{V_{2}} \neq 0$, i.e., there is some $v \in V_{2}$ such that $\langle u, v\rangle \neq 0$, hence $\langle v, u\rangle \neq 0$, which is equivalent to $\alpha_{21} \neq 0$. Let us now prove that $\left.\langle\cdot, \cdot\rangle\right|_{V_{1} \oplus V_{2}}$ is nondegenerate. Let $u+v \in V_{1} \oplus V_{2}$ and $\langle u+v, x+y\rangle=0$ for any $x+y \in V_{1} \oplus V_{2}$. We have $\langle u+v, x+y\rangle=\langle u, y\rangle+\langle v, x\rangle=0$, because $\left\langle V_{1}, V_{1}\right\rangle=\left\langle V_{2}, V_{2}\right\rangle=0$. Take $x=0$ in this equation; then $\langle u, y\rangle=0$ for any $y \in V_{2}$, hence $u=0$, because $\alpha_{12}\left(V_{1}\right)=\tilde{V}_{2}$. Analogously, $v=0$. Since $U$ is minimal, the same argument as above implies that $U=V_{1} \oplus V_{2} \cong V_{1} \oplus \tilde{V}_{1}$.

Proof of Proposition 1.6. By Lemma A.2

$$
\lambda \cong \nu \oplus \nu^{*} \oplus \lambda_{1}^{z_{1}} \oplus \cdots \oplus \lambda_{t}^{z_{t}},
$$

where $\nu$ is a representation of $G$ and $\lambda_{1}, \ldots, \lambda_{t}$ are pairwise nonisomorphic irreducible symplectic representations of $G$. Let $\nu=\nu_{1}^{l_{1}} \oplus \cdots \oplus \nu_{r}^{l_{r}}$, where $\nu_{1}, \ldots, \nu_{r}$ 
are pairwise nonisomorphic irreducible representations of $G$. By Lemma A.1 for each $i$ we have $\nu_{i}=\nu_{i}^{0} \otimes \phi_{i}$, where $\phi_{i}$ is a one-dimensional representation of $G$ and $\nu_{i}^{0}$ is an irreducible representation of $G$ trivial on $\left(c^{s}\right)$. It follows that $\nu_{i}^{0}$ can be considered as an irreducible representation of $H=G /\left\langle c^{2 s}\right\rangle$, and as such can be written in the form $\nu_{i}^{0}=\operatorname{Ind}_{E \rtimes \Gamma_{i}}^{H} \psi_{i}$, where $\psi_{i}$ is a one-dimensional representation of $E \rtimes \Gamma_{i}, \psi_{i}(e)=\xi_{i}$ for an $n$-th root of unity $\xi_{i}$ of order $d_{i}, x_{i}=|k|$ in $\left(\mathbb{Z} / d_{i} \mathbb{Z}\right)^{\times}$, and $\Gamma_{i}=\left\langle c^{x_{i}}\right\rangle$ (8), p. 62, Prop. 25). Thus

$$
\begin{gathered}
\nu_{i}^{0}(e)=\left(\begin{array}{cccc}
\xi_{i} & 0 & \ldots & 0 \\
0 & \xi_{i}^{k} & \ldots & 0 \\
\vdots & \vdots & \ddots & \vdots \\
0 & 0 & \ldots & \xi_{i}^{k^{x_{i}-1}}
\end{array}\right), \\
\nu_{i}(e)=\nu_{i}^{0} \otimes \phi_{i}(e)=\left(\begin{array}{cccc}
\xi_{i} \phi_{i}(e) & 0 & \ldots & 0 \\
0 & \left(\xi_{i} \phi_{i}(e)\right)^{k} & \ldots & 0 \\
\vdots & \vdots & \ddots & \vdots \\
0 & 0 & \ldots & \left(\xi_{i} \phi_{i}(e)\right)^{k^{x_{i}-1}}
\end{array}\right) .
\end{gathered}
$$

In the second matrix we used the relation $\phi_{i}(e)^{k-1}=1$, which follows from the fact that $\phi_{i}$ is a one-dimensional representation of $G$ and $c^{-1} e c=e^{k}$. By Proposition 1.5 each $\lambda_{i}$ can be considered as a representation of $H$ and $\lambda_{i}=\operatorname{Ind}_{E \rtimes L_{i}}^{H} \rho_{i}$, where $\rho_{i}$ is a one-dimensional representation of $E \rtimes L_{i}, \rho_{i}(e)=\eta_{i}$ for an $n$-th root of unity $\eta_{i}$ of order $u_{i}, y_{i}=|k|$ in $\left(\mathbb{Z} / u_{i} \mathbb{Z}\right)^{\times}, L_{i}=\left\langle c^{y_{i}}\right\rangle$, and $\rho_{i}\left(c^{y_{i}}\right)=-1$.

We will need the following lemma:

Lemma A.3. Let $d_{1}, \ldots, d_{m}$ be pairwise distinct natural numbers. For each $d_{i}$ let $p_{i}(X) \in \mathbb{C}[X]$ be a monic polynomial, all the roots of which are some primitive $d_{i}$-th roots of unity, and let $p(X)=p_{1}(X) \cdots p_{m}(X)$. If $p(X) \in \mathbb{Q}[X]$, then each $p_{i}(X)$ is a power of the $d_{i}$-th cyclotomic polynomial $\Phi_{d_{i}}(X)$.

Proof. The statement follows from considering the factorization of $p(X)$ into irreducibles in $\mathbb{Q}[X]$.

Since the characteristic polynomial $p$ of $\lambda(e)$ has coefficients in $\mathbb{Q}$, by Lemma A.3 we can assume that $\xi_{1} \phi_{1}(e), \ldots, \xi_{r} \phi_{r}(e), \eta_{1}, \ldots, \eta_{t}$ are primitive roots of unity of the same order $d$ and that $p=\Phi_{d}^{v}$ for some $v$, where $\Phi_{d}$ is the $d$-th cyclotomic polynomial. Indeed, $\lambda$ can be written as a sum of semisimple symplectic representations of $G$ which have this property, and it is enough to show that for each of them (1.5) holds.

Let $x=|k|$ in $(\mathbb{Z} / d \mathbb{Z})^{\times}$and $\Gamma=\left\langle c^{x}\right\rangle$. If $\lambda \cong \nu \oplus \nu^{*}$, then there is nothing to prove. Thus, we assume that there is $\lambda_{1}$ in A.5. Since $\lambda_{1}$ is symplectic, $x$ is even, $d \neq 1,2$, and $k^{\frac{x}{2}} \equiv-1(\bmod d)$ by Proposition 1.5 . Note that $x$ divides each $x_{i}$. Indeed, $\left(\xi_{i} \phi_{i}(e)\right)^{k^{x_{i}}}=\xi_{i} \phi_{i}(e)$, hence $k^{x_{i}} \equiv 1(\bmod d)$, because $\xi_{i} \phi_{i}(e)$ is a primitive $d$-th root of unity by assumption. For each $i$ denote by $p_{\nu_{i}}$ the characteristic polynomial of $\nu_{i}(e)$ and by $p_{\nu_{i}^{*}}$ the characteristic polynomial of $\nu_{i}^{*}(e)$. Then $p_{\nu_{i}}=p_{\nu_{i}^{*}}$. This is true because $x$ divides $x_{i}, x$ is even, $k^{\frac{x_{i}}{2}} \not \equiv 1(\bmod d)$, and $k^{\frac{x}{2}} \equiv-1(\bmod d)$; hence each root of $p_{\nu_{i}}$ appears in $p_{\nu_{i}}$ with its complex conjugate. Thus

$$
p=p_{\nu_{1}}^{2 l_{1}} \cdots p_{\nu_{r}}^{2 l_{r}} p_{\lambda_{1}}^{z_{1}} \cdots p_{\lambda_{t}}^{z_{t}}
$$

where for each $i$ we denote by $p_{\lambda_{i}}$ the characteristic polynomial of $\lambda_{i}(e)$. 
For each primitive $d$-th root of unity $\xi$ write $q(\xi)=(X-\xi)\left(X-\xi^{k}\right) \cdots\left(X-\xi^{k^{x-1}}\right)$, where $x=|k|$ in $(\mathbb{Z} / d \mathbb{Z})^{\times}$. Clearly, all $\xi, \xi^{k}, \ldots, \xi^{k^{x-1}}$ are distinct, and for two primitive $d$-th roots of unity $\xi$ and $\xi^{\prime}$ either $q(\xi)=q\left(\xi^{\prime}\right)$ or $q(\xi)$ and $q\left(\xi^{\prime}\right)$ have no common roots. In this notation $p_{\lambda_{i}}=q\left(\eta_{i}\right)$ and $p_{\nu_{i}}=q\left(\xi_{i} \phi_{i}(e)\right)^{\alpha_{i}}$, where $\alpha_{i}=\frac{x_{i}}{x}$. Since $\lambda_{1}, \ldots, \lambda_{t}$ are irreducible, symplectic, and pairwise nonisomorphic, it follows from Proposition 1.5 that $q\left(\eta_{i}\right) \neq q\left(\eta_{j}\right)$ for $i \neq j$. Without loss of generality we can assume that $p$ has the following form:

$$
p=q\left(\xi_{1} \phi_{1}(e)\right)^{2 m_{1}} \cdots q\left(\xi_{f} \phi_{f}(e)\right)^{2 m_{f}} q\left(\eta_{1}\right)^{z_{1}} \cdots q\left(\eta_{t}\right)^{z_{t}},
$$

where $f \leq r, m_{1}, \ldots, m_{f}$ are positive integers, and $q\left(\xi_{1} \phi_{1}(e)\right), \ldots, q\left(\xi_{f} \phi_{f}(e)\right)$ have no common roots. There are two possibilities:

(1) there exists some $q\left(\xi_{i} \phi_{i}(e)\right)$ which is not equal to any of $q\left(\eta_{1}\right), \ldots, q\left(\eta_{t}\right)$. Without loss of generality we can assume that $i=1$;

(2) each $q\left(\xi_{i} \phi_{i}(e)\right)$ equals some $q\left(\eta_{j}\right)$

Proof of Case (1). In this case, since $p=\Phi_{d}^{v}$, it follows from (A.6) that for each $j$ we have $z_{j}+2 \cdot \alpha(j)=2 m_{1}$, where $\alpha(j)=m_{\beta}$ if $q\left(\eta_{j}\right)$ equals some $q\left(\xi_{\beta} \phi_{\beta}(e)\right)$ and $\alpha(j)=0$ otherwise. Thus, in this case all $z_{1}, \ldots, z_{t}$ are even and $[\lambda]=[\nu]+$ $\left[\nu^{*}\right]+2 \cdot\left[\mu_{0}\right]$, where $\mu_{0}=\lambda_{1}^{\frac{z_{1}}{2}} \oplus \cdots \oplus \lambda_{t}^{\frac{z_{t}}{2}}$ is symplectic of finite image because all $\lambda_{1}, \ldots, \lambda_{t}$ are symplectic of finite images.

Proof of Case (2). In this case, since $p=\Phi_{d}^{v}$, it follows from (A.6) that for each $j$ we have $z_{j}+2 \cdot \alpha(j)=v$, where $\alpha(j)=m_{\beta}$ if $q\left(\eta_{j}\right)$ equals some $q\left(\xi_{\beta} \phi_{\beta}(e)\right)$ and $\alpha(j)=0$ otherwise. Moreover, it follows that $q\left(\eta_{1}\right) \cdots q\left(\eta_{t}\right)=\Phi_{d}$. Thus

$$
[\lambda]=[\nu]+\left[\nu^{*}\right]-2 \cdot\left[\mu_{0}^{\prime}\right]+v \cdot\left[\lambda_{1}\right]+\cdots+v \cdot\left[\lambda_{t}\right],
$$

where $\mu_{0}^{\prime}=\lambda_{1}^{\alpha(1)} \oplus \cdots \oplus \lambda_{t}^{\alpha(t)}$ is symplectic of finite image and it is enough to show that $\hat{\lambda}_{1} \oplus \cdots \oplus \hat{\lambda}_{t}$ is realizable over $\mathbb{Q}$. Recall that for each $i, \hat{\lambda}_{i}=\operatorname{Ind}_{E \rtimes \Gamma}^{H} \varphi_{i}$, where $\varphi_{i}(e)=\xi_{i}$ for some primitive $d$-th root of unity $\xi_{i}, x=|k|$ in $(\mathbb{Z} / d \mathbb{Z})^{\times}, \Gamma=\left\langle c^{x}\right\rangle$, and $\varphi_{i}\left(c^{x}\right)=1$ (see Proposition 1.5 and (1.4)). Since the representations of this form are completely defined by a root of unity $\xi$, we will denote them by $\Theta(\xi)$. For any $r$ dividing $d$ the cyclic group $\langle k\rangle$ acts on the set of all primitive $r$-th roots of unity via $\xi \longmapsto \xi^{k}$. Let $\left\{\xi_{r}^{1}, \ldots, \xi_{r}^{w_{r}}\right\}$ be the set of representatives for this action and let

$$
\Theta(r)=\bigoplus_{i=1}^{w_{r}} \Theta\left(\xi_{r}^{i}\right)
$$

Then the characteristic polynomial of $\Theta(r)(e)$ is just $\Phi_{r}$. Since the characteristic polynomial of $\left(\hat{\lambda}_{1} \oplus \cdots \oplus \hat{\lambda}_{t}\right)(e)$ is $q\left(\eta_{1}\right) \cdots q\left(\eta_{t}\right)=\Phi_{d}$, it follows that $\hat{\lambda}_{1} \oplus \cdots \oplus \hat{\lambda}_{t}=$ $\Theta(d)$. By induction on $d$ we will prove that each $\Theta(d)$ is realizable over $\mathbb{Q}$.

Clearly, $\Theta(d)$ is realizable over $\mathbb{Q}$ when $d=1$, because in this case $\Theta(d)=1$. Let $L=\left\langle e^{d}\right\rangle \rtimes C$ and $\pi=\operatorname{Ind}_{L}^{H} 1$. Then the characteristic polynomial of $\pi(e)$ is $x^{d}-1$, consequently,

$$
\pi \cong \bigoplus_{r \mid d} \Theta(r) \quad \text { on } \quad E .
$$

We will prove that this is true on the whole group $H$. Observe, that for any $r$ all $\Theta\left(\xi_{r}^{1}\right), \ldots, \Theta\left(\xi_{r}^{w_{r}}\right)$ are irreducible over $\mathbb{C}$ and $\Theta\left(\xi_{r}^{i}\right) \cong \Theta\left(\xi_{r^{\prime}}^{i^{\prime}}\right)$ only if $i=i^{\prime}$ and 
$r=r^{\prime}$. Let $\chi_{r}^{i}$ be the character of $\Theta\left(\xi_{r}^{i}\right)$. Then, using Frobenius reciprocity, we have

$$
\begin{aligned}
\left\langle\pi, \Theta\left(\xi_{r}^{i}\right)\right\rangle & =\left\langle\operatorname{Ind}_{L}^{H} 1, \Theta\left(\xi_{r}^{i}\right)\right\rangle=\left\langle 1, \operatorname{Res}_{L}^{H} \Theta\left(\xi_{r}^{i}\right)\right\rangle \\
& =\frac{d}{2 n s} \sum_{u, v} \chi_{r}^{i}\left(e^{d u} c^{v}\right)=\frac{d}{2 n s} \sum_{u, v} \chi_{r}^{i}\left(c^{v}\right)=1,
\end{aligned}
$$

hence $\pi \cong \bigoplus_{r \mid d} \Theta(r)$ on $H$. Since $\pi$ is realizable over $\mathbb{Q}$ and $\Theta(r)$ is realizable over $\mathbb{Q}$ for any $r<d$ by induction, $\Theta(d)=\pi-\bigoplus_{r \mid d, r \neq d} \Theta(r)$ is realizable over $\mathbb{Q}$.

Proof of Proposition 1.9, Let $\lambda=\kappa \otimes \omega^{1 / 2}$. Then $W(\kappa \otimes \tau)=W(\lambda \otimes \tau)$, because real powers of $\omega$ do not change the root number. Since the root number of representations of $\mathcal{W}(\bar{K} / K)$ is multiplicative in short exact sequences, there is a unique homomorphism

$$
\alpha: R(\mathcal{W}(\bar{K} / K)) \longrightarrow \mathbb{C}^{\times}
$$

such that $\alpha([\lambda])=W(\lambda)$ for any representation $\lambda$ of $\mathcal{W}(\bar{K} / K)$. Thus, it follows from Corollary 1.7 that

$$
W(\lambda \otimes \tau)=W(\mu \otimes \tau) \cdot W\left(\mu^{*} \otimes \tau\right) \cdot \frac{W\left(\mu_{0} \otimes \tau\right)^{2}}{W\left(\mu_{0}^{\prime} \otimes \tau\right)^{2}} \cdot W\left(\mu_{1} \otimes \tau\right) \cdots W\left(\mu_{a} \otimes \tau\right) .
$$

Since $\tau$ has finite image and real-valued character, we have

$$
\begin{aligned}
W(\mu \otimes \tau) \cdot W\left(\mu^{*} \otimes \tau\right) & =W(\mu \otimes \tau) \cdot W\left((\mu \otimes \tau)^{*}\right) \\
& =\operatorname{det}(\mu \otimes \tau)(-1)=\operatorname{det} \mu(-1)^{\operatorname{dim} \tau} \cdot \operatorname{det} \tau(-1)^{\operatorname{dim} \mu} .
\end{aligned}
$$

Also, since $\mu_{0}$ and $\mu_{0}^{\prime}$ are symplectic and of finite images, $W\left(\mu_{0} \otimes \tau\right)= \pm 1$ and $W\left(\mu_{0}^{\prime} \otimes \tau\right)= \pm 1($ [7, p. 315), hence from (A.7) we get

$$
W(\lambda \otimes \tau)=\operatorname{det} \mu(-1)^{\operatorname{dim} \tau} \cdot \operatorname{det} \tau(-1)^{\operatorname{dim} \mu} \cdot W\left(\mu_{1} \otimes \tau\right) \cdots W\left(\mu_{a} \otimes \tau\right) .
$$

Thus, we need to compute $W\left(\mu_{1} \otimes \tau\right), \ldots, W\left(\mu_{a} \otimes \tau\right)$. Let $\gamma$ be an irreducible symplectic subrepresentation of $\lambda$. Let $L / K^{u n r}$ be a minimal subextension of $\bar{K} / K^{u n r}$ over which $B$ acquires good reduction. Then, as was discussed in Subsection 1.2. $\lambda$ and, consequently, $\gamma$ can be considered as representations of $G=E \rtimes\langle\Phi\rangle$, where $E=\operatorname{Gal}\left(L / K^{u n r}\right)$ is a finite cyclic group (because $p>2 m+1$ ) and $\langle\Phi\rangle$ is an infinite cyclic group. Let $x=\operatorname{dim} \gamma$. Then by Proposition 1.5 as a representation of $G, \gamma$ is induced from a one-dimensional representation of $E \rtimes\left\langle\Phi^{x}\right\rangle$. Hence, as a representation of $\mathcal{W}(\bar{K} / K), \gamma$ is induced from a one-dimensional representation $\phi$ of $\mathcal{W}\left(\bar{K} / H_{x}\right)$, where $H_{x}$ is the unramified extension of $K$ of degree $x$, i.e., $\gamma=\operatorname{Ind}_{K}^{H_{x}} \phi$. Since $\gamma$ is symplectic, $x$ is even. Let $x=2 y$ and let $H_{y}$ be the unramified extension of $K$ of degree $y$, hence $K \subseteq H_{y} \subseteq H_{x}$. Let $\gamma^{\prime}=\operatorname{Ind}_{H_{y}}^{H_{x}} \phi, \tau^{\prime}=\operatorname{Res}_{K}^{H_{y}} \tau$; then $\gamma=\operatorname{Ind}_{K}^{H_{y}} \gamma^{\prime}$ and by Formula (1.4) on p. 316 of [7] we have

$$
W(\gamma \otimes \tau)=W\left(\operatorname{Ind}_{K}^{H_{y}}\left(\gamma^{\prime} \otimes \tau^{\prime}\right)\right)=W\left(\gamma^{\prime} \otimes \tau^{\prime}\right) W\left(\operatorname{Ind}_{K}^{H_{y}} 1_{H_{y}}\right)^{2 \operatorname{dim} \tau} .
$$

Let us first prove that $W\left(\operatorname{Ind}_{K}^{H_{y}} 1_{H_{y}}\right)^{2 \operatorname{dim} \tau}=1$. Let $\varpi$ be a uniformizer of $K$. It is easy to check that $\operatorname{Ind}_{K}^{H_{y}} 1_{H_{y}}=\bigoplus_{i=0}^{y-1} \chi_{i}$, where $\chi_{0}, \ldots, \chi_{y-1}$ are all the distinct unramified characters of $K^{\times}$satisfying $\chi_{i}(\varpi)^{y}=1$. Hence $W\left(\operatorname{Ind}_{K}^{H_{y}} 1_{H_{y}}\right)=$ $\prod_{i=0}^{y-1} W\left(\chi_{i}\right)$. By Formula $(\epsilon 3)$ on p. 142 of [6] for each $i$ we have $W\left(\chi_{i}\right)=\xi_{i}^{n(\psi)}$, 
where $n(\psi) \in \mathbb{Z}$, each $\xi_{i}$ is a $y$-th root of unity, and $\xi_{i} \neq \xi_{j}$ if $i \neq j$. Hence $\prod_{i=0}^{y-1} W\left(\chi_{i}\right)=\prod_{i=0}^{y-1} \xi_{i}^{n(\psi)}= \pm 1$ and

$$
W\left(\operatorname{Ind}_{K}^{H_{y}} 1_{H_{y}}\right)^{2 \operatorname{dim} \tau}=1 .
$$

To compute $W\left(\gamma^{\prime} \otimes \tau^{\prime}\right)$ we will show that Theorem 1.8 can be applied to $H_{y}$, $\gamma^{\prime}$, and $\tau^{\prime}$. Indeed, $\tau^{\prime}$ is a representation of $\operatorname{Gal}\left(\bar{K} / H_{y}\right)$ with real-valued character and $\gamma^{\prime}=\operatorname{Ind}_{H_{y}}^{H_{x}} \phi$ is a two-dimensional representation of $\mathcal{W}\left(\bar{K} / H_{y}\right)$ induced from a character $\phi$ of finite image (by Proposition 1.5), hence $\gamma^{\prime}$ is a representation of $\operatorname{Gal}\left(\bar{K} / H_{y}\right)$. Since $\operatorname{Ind}_{K}^{H_{y}} \gamma^{\prime}=\gamma$ is irreducible, $\gamma^{\prime}$ is irreducible too. Since $\operatorname{dim} \gamma^{\prime}=$ $2, \gamma^{\prime}$ is symplectic if and only if $\operatorname{det} \gamma^{\prime}$ is trivial, because $\operatorname{Sp}(2, \mathbb{C})=\operatorname{SL}(2, \mathbb{C})([7$, p. 317). From Proposition 1.5 we find that as a representation of $E \rtimes\left\langle\Phi^{y}\right\rangle$, $\gamma^{\prime}$ has the form

$$
\gamma^{\prime}(e)=\left(\begin{array}{cc}
\xi & 0 \\
0 & \xi^{-1}
\end{array}\right), \quad \gamma^{\prime}\left(\Phi^{y}\right)=\left(\begin{array}{cc}
0 & -1 \\
1 & 0
\end{array}\right),
$$

where $e$ is a generator of $E, \xi$ is a root of unity. It follows immediately that $\operatorname{det} \gamma^{\prime}=1$. Thus to be able to apply Theorem 1.8, we need only to check that $\phi$ is a tame character of $H_{x}^{\times}$. This follows from the fact that $\phi$ is trivial on $\operatorname{Gal}(\bar{K} / L)$ and $L / K^{u n r}$ is tamely ramified, because $p>2 m+1$. By Theorem 1.8

$$
W\left(\gamma^{\prime} \otimes \tau^{\prime}\right)=\operatorname{det} \tau^{\prime}(-1) \cdot \varphi^{\operatorname{dim} \tau} \cdot(-1)^{\left\langle 1, \tau^{\prime}\right\rangle+\left\langle\eta^{\prime}, \tau^{\prime}\right\rangle+\left\langle\hat{\gamma}^{\prime}, \tau^{\prime}\right\rangle},
$$

where $\eta^{\prime}$ is the unramified quadratic character of $H_{y}^{\times}, \hat{\gamma}^{\prime}=\operatorname{Ind}_{H_{y}}^{H_{x}}(\phi \otimes \theta), \theta$ is the unramified quadratic character of $H_{x}^{\times}$, and $\varphi= \pm 1$.

Since $\tau^{\prime}=\operatorname{Res}_{K}^{H_{y}} \tau$, we have $\operatorname{det} \tau^{\prime}=\operatorname{det} \tau \circ N_{H_{y} / K}$, hence

$$
\operatorname{det} \tau^{\prime}(-1)=\operatorname{det} \tau(-1)^{\left[H_{y}: K\right]}=\operatorname{det} \tau(-1)^{y} .
$$

By Frobenius reciprocity

$$
\left\langle 1, \tau^{\prime}\right\rangle+\left\langle\eta^{\prime}, \tau^{\prime}\right\rangle=\left\langle 1_{H_{y}} \oplus \eta^{\prime}, \tau^{\prime}\right\rangle=\left\langle 1_{H_{y}} \oplus \eta^{\prime}, \operatorname{Res}_{K}^{H_{y}} \tau\right\rangle=\left\langle\operatorname{Ind}_{K}^{H_{y}}\left(1_{H_{y}} \oplus \eta^{\prime}\right), \tau\right\rangle .
$$

As was mentioned above, $\operatorname{Ind}_{K}^{H_{y}} 1_{H_{y}}=\bigoplus_{i=0}^{y-1} \chi_{i}$, where $\chi_{0}, \ldots, \chi_{y-1}$ are all the distinct unramified characters of $K^{\times}$satisfying $\chi_{i}(\varpi)^{y}=1$. Analogously, $\operatorname{Ind}_{K}^{H_{y}} \eta^{\prime}=$ $\bigoplus_{i=y}^{2 y-1} \chi_{i}$, where $\chi_{y}, \ldots, \chi_{2 y-1}$ are all the distinct unramified characters of $K^{\times}$ satisfying $\chi_{i}^{y}(\varpi)=-1(y \leq i \leq 2 y-1)$. Thus

$$
\operatorname{Ind}_{K}^{H_{y}}\left(1_{H_{y}} \oplus \eta^{\prime}\right)=\bigoplus_{i=0}^{2 y-1} \chi_{i}
$$

where $\chi_{0}, \ldots, \chi_{2 y-1}$ are all the distinct unramified characters of $K^{\times}$satisfying $\chi_{i}(\varpi)^{2 y}=1$; hence

$$
\left\langle 1, \tau^{\prime}\right\rangle+\left\langle\eta^{\prime}, \tau^{\prime}\right\rangle=\sum_{i=0}^{2 y-1}\left\langle\chi_{i}, \tau\right\rangle .
$$

Since $\tau$ has a real-valued character, for each $\chi_{i}$ of order greater than $2,\left\langle\chi_{i}, \tau\right\rangle$ will appear in this sum twice, i.e.,

$$
\left\langle 1, \tau^{\prime}\right\rangle+\left\langle\eta^{\prime}, \tau^{\prime}\right\rangle \equiv\langle 1, \tau\rangle+\langle\eta, \tau\rangle \quad(\bmod 2) .
$$

Finally, by Frobenius reciprocity,

$$
\left\langle\hat{\gamma}^{\prime}, \tau^{\prime}\right\rangle=\left\langle\operatorname{Ind}_{H_{y}}^{H_{x}}(\phi \otimes \theta), \operatorname{Res}_{K}^{H_{y}} \tau\right\rangle=\left\langle\operatorname{Ind}_{K}^{H_{x}}(\phi \otimes \theta), \tau\right\rangle=\langle\hat{\gamma}, \tau\rangle .
$$


Now formulas (A.9) - A.14 imply

$$
W(\gamma \otimes \tau)=\operatorname{det} \tau(-1)^{y} \cdot \varphi^{\operatorname{dim} \tau} \cdot(-1)^{\langle 1, \tau\rangle+\langle\eta, \tau\rangle+\langle\hat{\gamma}, \tau\rangle} .
$$

Applying (A.15) to $\mu_{1}, \ldots, \mu_{a}$ and substituting the result into (A.8) we get the statement of the proposition.

\section{Appendix B.}

Lemma B.1. Let $G$ be a semi-abelian scheme over a field $K$. Then $G(\bar{K})$ is a divisible abelian group.

Proof. The statement follows from the definition of a semi-abelian scheme together with the fact that the groups of points over $\bar{K}$ of a torus or of an abelian variety are divisible.

In the next lemma we keep the notation of Subsection 1.1.

Lemma B.2. Let $\sigma^{\prime}=(\sigma, N)$ be the representation of $\mathcal{W}^{\prime}(\bar{K} / K)$ associated to the natural l-adic representation of $\operatorname{Gal}(\bar{K} / K)$ on $V_{l}(A)^{*}$ and let $\sigma^{\prime} \cong \gamma \oplus(\delta \otimes \operatorname{sp}(2))$ for some representations $\gamma$ and $\delta$ of $\mathcal{W}(\bar{K} / K)$. Then $\operatorname{dim} \delta=r$.

Proof. Since $\operatorname{dim} \delta=\operatorname{rank} N$ and for any finite extension $L \subset \bar{K}$ of $K$ we have $\operatorname{Res}_{\mathcal{W}^{\prime}(\bar{K} / L)}^{\mathcal{W}^{\prime}(\bar{K} / K)} \sigma^{\prime}=\left(\operatorname{Res}_{K}^{L} \sigma, N\right)([\underline{6}$, p. 130), we can assume that $T$ splits over $K$ and $B$ has good reduction over $K$.

We have the following exact sequence of $\operatorname{Gal}(\bar{K} / K)$-modules $([4$, p. 312):

$$
0 \longrightarrow G(\bar{K})_{l^{n}} \longrightarrow A(\bar{K})_{l^{n}} \stackrel{\phi_{l^{n}}}{\longrightarrow} Y(\bar{K}) / l^{n} Y(\bar{K}) \longrightarrow 0
$$

Since $G(\bar{K})$ is divisible by Lemma B.1, sequence (B.1) induces an exact $\mathrm{Gal}(\bar{K} / K)$ equivariant sequence of $l$-adic Tate modules:

$$
0 \longrightarrow T_{l}(G) \longrightarrow T_{l}(A) \longrightarrow Z \longrightarrow 0
$$

where $Z=\lim _{(}\left(Y(\bar{K}) / l^{n} Y(\bar{K})\right)$ with the maps being the natural quotient maps. By tensoring the above sequence with $\mathbb{Q}_{l}$ over $\mathbb{Z}_{l}$ we get the following exact $\operatorname{Gal}(\bar{K} / K)$ equivariant sequence:

$$
0 \longrightarrow V_{l}(G) \longrightarrow V_{l}(A) \stackrel{\phi}{\longrightarrow} Z \otimes_{\mathbb{Z}_{l}} \mathbb{Q}_{l} \longrightarrow 0 .
$$

For a positive integer $n$ let $\mu_{n}$ denote the group of $n$-th roots of unity in $\bar{K}$, and $T_{l}(\mu)=\lim \mu_{l^{n}}$ with the $l$-th power maps. Let $K_{l^{n}}$ be the tamely ramified extension of $\overleftarrow{K^{u} n r}$ of degree $l^{n}$ and let $t_{l^{n}}: I \longrightarrow \mu_{l^{n}}$ be the composition of the restriction map onto $\operatorname{Gal}\left(K_{l^{n}} / K^{u n r}\right)$ with the isomorphism $\operatorname{Gal}\left(K_{l^{n}} / K^{u n r}\right) \cong \mu_{l^{n}}$.

Let $x_{l^{n}} \in A(\bar{K})_{l^{n}}, i \in I$, and $\phi_{l^{n}}\left(x_{l^{n}}\right)=[y]$ for some $y \in Y(\bar{K})$ and $\phi_{l^{n}}$ given by (B.1). Then a formula on p. 314 of [4] yields:

$$
i\left(x_{l^{n}}\right)=x_{l^{n}}+\nu_{l^{n}}\left(y \otimes t_{l^{n}}(i)\right)
$$

where $\nu_{l^{n}}: Y(\bar{K}) \otimes_{\mathbb{Z}} \mu_{l^{n}} \longrightarrow T(\bar{K})_{l^{n}}$ is the following composition of $\operatorname{Gal}(\bar{K} / K)$ module homomorphisms:

$$
Y(\bar{K}) \otimes_{\mathbb{Z}} \mu_{l^{n}} \longrightarrow \operatorname{Hom}_{\mathbb{Z}}(X(\bar{K}), \mathbb{Z}) \otimes_{\mathbb{Z}} \mu_{l^{n}} \stackrel{\sim}{\longrightarrow} T(\bar{K})_{l^{n}},
$$

where $X$ is the character group of $T$ and the first map is induced by the geometric monodromy

$$
\mu_{0}: Y \times X \longrightarrow \mathbb{Z}
$$


finally, $\nu_{l^{n}}\left(y \otimes t_{l^{n}}(i)\right) \in T(\bar{K})_{l^{n}}$ is considered as an element of $A(\bar{K})_{l^{n}}$ via the inclusions

$$
T(\bar{K})_{l^{n}} \hookrightarrow G(\bar{K})_{l^{n}} \hookrightarrow A(\bar{K})_{l^{n}} .
$$

For each $i \in I$ we have the maps $\alpha_{n}(i): Y(\bar{K}) / l^{n} Y(\bar{K}) \longrightarrow T(\bar{K})_{l^{n}}$ given by the following composition:

$$
Y(\bar{K}) / l^{n} Y(\bar{K}) \stackrel{\psi_{n}(i)}{\longrightarrow} Y(\bar{K}) \otimes_{\mathbb{Z}} \mu_{l^{n}} \stackrel{\nu_{l^{n}}}{\longrightarrow} T(\bar{K})_{l^{n}},
$$

where $\psi_{n}(i)([y])=y \otimes t_{l^{n}}(i), y \in Y(\bar{K})$. It is easy to show that $\left\{\alpha_{n}(i)\right\}$ induces the homomorphism

$$
\alpha(i)=\left(\alpha_{n}(i)\right): Z \longrightarrow T_{l}(T),
$$

where $Z=\lim _{\longleftarrow}\left(Y(\bar{K}) / l^{n} Y(\bar{K})\right)$. By extending scalars to $\mathbb{Q}_{l}$ we get

$$
\alpha^{\prime}(i): Z \otimes_{\mathbb{Z}_{l}} \mathbb{Q}_{l} \longrightarrow V_{l}(T) .
$$

Let $\beta_{l}: \operatorname{Gal}(\bar{K} / K) \longrightarrow \mathrm{GL}\left(V_{l}(A)\right)$ be the natural $l$-adic representation of

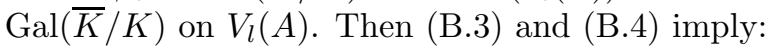

$$
\beta_{l}(i)=\mathrm{id}+\alpha^{\prime}(i) \circ \phi, \quad i \in I,
$$

where id $: V_{l}(A) \longrightarrow V_{l}(A)$ is the identity map and $\phi$ is given by (B.2). On the other hand,

$$
\beta_{l}(i)=\exp \left(a_{l}(i) R_{l}\right)
$$

where $i$ is in some open subgroup $J$ of $I, a_{l}: I \longrightarrow \mathbb{Q}_{l}$ is a nontrivial continuous homomorphism, and $R_{l}$ is a nilpotent endomorphism on $V_{l}(A)$ ([6], Prop. on p. 131). Since $\sigma^{\prime}=(\sigma, N)$ is the representation of $\mathcal{W}^{\prime}(\bar{K} / K)$ associated to $\beta_{l}^{*}$ : $\operatorname{Gal}(\bar{K} / K) \longrightarrow \mathrm{GL}\left(V_{l}(A)^{*}\right)$, it follows that $N$ is obtained from $-R_{l}^{t}$ by extending scalars via a field embedding $\imath: \mathbb{Q}_{l} \hookrightarrow \mathbb{C}$. Thus, $\operatorname{rank} R_{l}=\operatorname{rank} N=\operatorname{dim} \delta$ and $R_{l}^{2}=N^{2}=0$ by assumption. Thus,

$$
\alpha^{\prime}(i) \circ \phi=a_{l}(i) R_{l}, \quad i \in J
$$

and, since $\phi$ is surjective, it is enough to show that there exists $i_{0} \in I$ such that $\alpha^{\prime}\left(i_{0}\right)$ is surjective.

There exists $i_{0} \in I$ such that $t_{l^{n}}\left(i_{0}\right)$ is a generator of $\mu_{l^{n}}$ for each $n$. This implies that $\psi_{n}\left(i_{0}\right)$ is an isomorphism for each $n$, hence it is enough to show that the map

$$
\nu^{\prime}: \lim _{\longleftarrow}\left(Y(\bar{K}) \otimes_{\mathbb{Z}} \mu_{l^{n}}\right) \otimes_{\mathbb{Z}_{l}} \mathbb{Q}_{l} \longrightarrow V_{l}(T)
$$

induced by $\left(\nu_{l^{n}}\right)$ is surjective. Since $\mu_{0}$ is nondegenerate ([2], p. 52, Remark 6.3), we have the following exact sequence:

$$
0 \longrightarrow Y(\bar{K}) \stackrel{g}{\longrightarrow} \operatorname{Hom}_{\mathbb{Z}}(X(\bar{K}), \mathbb{Z}) \longrightarrow M \longrightarrow 0,
$$

where $g(y)=\mu_{0}(y, \cdot)$ and $M$ is finite, since $Y(\bar{K})$ and $\operatorname{Hom}_{\mathbb{Z}}(X(\bar{K}), \mathbb{Z})$ are free abelian groups of the same rank $r$. Applying the functor $(-) \otimes_{\mathbb{Z}} \mu_{l^{n}}$ to the above sequence, we get:

$$
Y(\bar{K}) \otimes_{\mathbb{Z}} \mu_{l^{n}} \stackrel{\nu_{l n}}{\longrightarrow} T(\bar{K})_{l^{n}} \longrightarrow M \otimes_{\mathbb{Z}} \mu_{l^{n}} \longrightarrow 0,
$$

hence we have the exact sequence

$$
0 \longrightarrow \operatorname{im} \nu_{l^{n}} \longrightarrow T(\bar{K})_{l^{n}} \longrightarrow M / l^{n} M \longrightarrow 0 .
$$


Since $Y(\bar{K}) \otimes_{\mathbb{Z}} \mu_{l^{n}}$ is a finite group, $\operatorname{im} \nu_{l^{n}}$ is a finite group, hence $\left\{\operatorname{im} \nu_{l^{n}}\right\}$ satisfies the Mittag-Leffler condition and we have the following exact sequence:

$$
0 \longrightarrow \underset{\lim }{\longleftarrow}\left(\operatorname{im} \nu_{l^{n}}\right) \longrightarrow T_{l}(T) \longrightarrow \lim \left(M / l^{n} M\right) \longrightarrow 0 .
$$

Here $\lim \left(\operatorname{im} \nu_{l^{n}}\right) \cong \operatorname{im} \nu$, where

$$
\nu=\left(\nu_{l^{n}}\right): \lim _{\longleftarrow}\left(Y(\bar{K}) \otimes_{\mathbb{Z}} \mu_{l^{n}}\right) \longrightarrow T_{l}(T) .
$$

Indeed, let $S_{n}=Y(\bar{K}) \otimes_{\mathbb{Z}} \mu_{l^{n}}$; then we have an exact sequence

$$
0 \longrightarrow \operatorname{ker} \nu_{l^{n}} \longrightarrow S_{n} \longrightarrow \operatorname{im} \nu_{l^{n}} \longrightarrow 0,
$$

where the maps from $S_{n}$ to im $\nu_{l^{n}}$ are induced by $\nu_{l^{n}}$. Since $\operatorname{ker} \nu_{l^{n}}$ is finite for each $n,\left\{\operatorname{ker} \nu_{l^{n}}\right\}$ satisfies the Mittag-Leffler condition, hence there is the following exact sequence:

$$
0 \longrightarrow \lim _{\longleftarrow}\left(\operatorname{ker} \nu_{l^{n}}\right) \longrightarrow \lim _{\longleftarrow} S_{n} \longrightarrow \lim _{\longleftarrow}\left(\operatorname{im} \nu_{l^{n}}\right) \longrightarrow 0
$$

which together with (B.5) implies $\lim \left(\operatorname{im} \nu_{l^{n}}\right) \cong \operatorname{im} \nu$.

Thus, applying the exact functor $(-) \otimes_{\mathbb{Z}_{l}} \mathbb{Q}_{l}$ to (B.5) and taking into account that $M$ is finite, we get:

$$
0 \longrightarrow(\mathrm{im} \nu) \otimes_{\mathbb{Z}_{l}} \mathbb{Q}_{l} \longrightarrow V_{l}(T) \longrightarrow 0,
$$

which implies

$$
\operatorname{im} \nu^{\prime} \cong(\operatorname{im} \nu) \otimes_{\mathbb{Z}_{l}} \mathbb{Q}_{l} \cong V_{l}(T)
$$

hence $\nu^{\prime}$ is surjective.

\section{ACKNOWLEDGEMENTS}

This paper has been extracted from my Ph.D. dissertation. I would like to thank my advisor, Ted Chinburg, for suggesting the problem and useful discussions, and David Rohrlich for his attention to this work. I am also grateful to Siegfried Bosch, Ching-Li Chai, Robert Kottwitz, and Michel Raynaud for answering my questions related to the uniformization theory, and especially to Michel Raynaud for providing the reference [4] and to Ching-Li Chai for noting that the results of the paper which were originally proved for Jacobian varieties can be applied to arbitrary abelian varieties. I am also thankful to Brian Conrad for his valuable comments.

Last but not least, I am deeply grateful to the referee for making several corrections to the paper and suggesting many improvements of the exposition.

\section{REFERENCES}

1. C. Chevalley, Théorie des groupes de Lie III (Hermann, Paris, 1955). MR0068552 (16:901a)

2. G. Faltings and C.-L. Chai, Degeneration of abelian varieties (Springer-Verlag, Berlin, 1990). MR $1083353(92 \mathrm{~d}: 14036)$

3. J. S. Milne, Abelian varieties, Arithmetic Geometry (Springer, New York, 1986), 103-150. MR0861974

4. M. Raynaud, 1-motifs et monodromie géométrique, Astérisque 223 (1994), 295-319. MR.1293976 (95h:14020)

5. D. E. Rohrlich, The vanishing of certain Rankin-Selberg convolutions, Automorphic Forms and Analytic Number Theory (Montréal, PQ, 1989), 123-133. MR1111015 (92d:11051)

6. D. E. Rohrlich, Elliptic curves and the Weil-Deligne group, Elliptic Curves and Related Topics (CRM Proc. Lecture Notes, 4, Amer. Math. Soc., Providence, RI, 1994), 125-157. MR1260960 (95a:11054) 
7. D. E. Rohrlich, Galois theory, elliptic curves, and root numbers, Compositio Math. 100 (1996), 311-349. MR 1387669 (97m:11075)

8. J.-P. Serre, Linear representations of finite groups (Springer-Verlag, New York-Heidelberg, 1977). MR0450380 (56:8675)

9. J.-P. Serre and J. Tate, Good reduction of abelian varieties, Ann. of Math. 88 (1968), 492-517. MR $0236190(38: 4488)$

10. J. Tate, Endomorphisms of abelian varieties over finite fields, Invent. Math. 2 (1966), 134144. MR0206004 (34:5829)

Department of Mathematics, University of Pennsylvania, Philadelphia, Pennsylvania 19104

E-mail address: sabitova@math.upenn.edu

Current address: Department of Mathematics, University of Illinois at Urbana-Champaign, Urbana, Illinois 61801

E-mail address: sabitova@math.uiuc.edu 